Pure and Applied Mathematics Quarterly

Volume 5, Number 4

(Special Issue: In honor of

John Tate, Part 1 of 2)

1253-1310, 2009

\title{
Codimensions of Root Valuation Strata
}

\author{
Mark Goresky, ${ }^{1}$ Robert Kottwitz ${ }^{2}$ and Robert MacPherson \\ Dedicated to John Tate.
}

\begin{abstract}
The set of integral regular semisimple elements in $\mathfrak{g}((\epsilon))$ can be partitioned into strata, called root valuation strata, and the same is true of the adjoint quotient of $\mathfrak{g}((\epsilon))$. The main result of this paper is a formula for the codimensions of these root valuation strata in the adjoint quotient.
\end{abstract}

Keywords: Adjoint quotient, root valuation strata, affine Springer fiber.

\section{INTRODUCTION}

The topic of this paper arises naturally in the context of affine Springer fibers, which we now take a moment to discuss. Let $G$ be a semisimple complex algebraic group, and let $\mathfrak{g}$ denote its Lie algebra. We then have the affine Grassmannian $X=G(F) / G(\mathcal{O})$, where $\mathcal{O}$ is the ring $\mathbb{C}[[\epsilon]]$ of formal power series, and $F$ is its fraction field $\mathbb{C}((\epsilon))$. For any $u \in \mathfrak{g}(F)=\mathfrak{g} \otimes_{\mathbb{C}} F$ the closed subset

$$
X^{u}=\left\{g \in G(F) / G(\mathcal{O}): \operatorname{Ad}(g)^{-1} u \in \mathfrak{g}(\mathcal{O})=\mathfrak{g} \otimes_{\mathbb{C}} \mathcal{O}\right\}
$$

of the affine Grassmannian, first studied by Kazhdan-Lusztig in [KL88], is called the affine Springer fiber associated to $u$.

Received August 15, 2006.

1991 Mathematics Subject Classification. Primary 11F85; Secondary 20G25, 22 E67.

${ }^{1}$ The author's research supported in part by NSF grant DMS-0139986 and DARPA grant HR001104-1-0031.

${ }^{2}$ The author's research supported in part by NSF grants DMS-0071971 and DMS-0245639 
We now assume that $u$ is regular semisimple and write $T_{u}$ for its centralizer in $G$, a maximal torus of $G$ over $F$. We will also need $A_{u}$, the maximal $F$-split subtorus of $T_{u}$. If $u$ is integral, in the sense that $\alpha(u)$ is integral over $\mathcal{O}$ for every root $\alpha$ of $T_{u}$, then $X^{u}$ is non-empty and may be viewed (see [KL88]) as the set of $\mathbb{C}$-points of a scheme locally of finite type over $\mathbb{C}$. The dimension formula of Bezrukavnikov-Kazhdan-Lusztig (see [KL88] and [Bez96]) states that

$$
\operatorname{dim} X^{u}=\left(\delta_{u}-c_{u}\right) / 2,
$$

where

$$
\begin{aligned}
\delta_{u} & :=\operatorname{val} \operatorname{det}\left(\operatorname{ad}(u) ; \mathfrak{g}(F) / \mathfrak{t}_{u}(F)\right), \\
c_{u} & :=\operatorname{dim} T_{u}-\operatorname{dim} A_{u} .
\end{aligned}
$$

Here val is the usual valuation on $F$, normalized so that $\operatorname{val}(\epsilon)=1$, and of course $\mathfrak{t}_{u}(F)$ denotes the Lie algebra of the $F$-torus $T_{u}$.

In particular $\operatorname{dim} X^{u}$ depends only on the discrete invariant $\left(\delta_{u}, c_{u}\right)$ of $u$. It is useful however to introduce a finer invariant, still discrete in nature. For this we need to choose an algebraic closure $\bar{F}$ of $F$. We denote by $\tau$ the unique element of $\operatorname{Gal}(\bar{F} / F)$ that multiplies each $m$-th root of $\epsilon$ by $\exp (2 \pi i / m)$. Recall that $\tau$ is a topological generator of $\operatorname{Gal}(\bar{F} / F)$ and allows us to identify that Galois group with the profinite completion of $\mathbb{Z}$.

Fix a maximal torus $T$ of $G$ over $\mathbb{C}$. We write $R$ for the set of roots of $T$ in $G$, and $W$ for the Weyl group of $T$. Choose an element $u^{\prime} \in \mathfrak{t}(\bar{F})$ that is $G(\bar{F})$-conjugate to $u$. We attach to $u^{\prime}$ a pair $(w, r)$ in the following way: $w$ is the unique element of $W$ such that $w \tau\left(u^{\prime}\right)=u^{\prime}$, and $r: R \rightarrow \mathbb{Q}$ is the function defined by $r(\alpha):=\operatorname{val} \alpha\left(u^{\prime}\right)$. Here we have extended our valuation on $F$ to one on $\bar{F}$; the valuation of any $m$-th root of $\epsilon$ is then $1 / m$. Since $u$ is integral, the function $r$ takes values in the set of non-negative rational numbers. The element $u^{\prime}$ is not quite well-defined, since it may be replaced by $x u^{\prime}$ for any $x \in W$. This replaces $(w, r)$ by $\left(x w x^{-1}, x r\right)$, where $(x r)(\alpha):=r\left(x^{-1} \alpha\right)$.

All in all, we have associated to $u$ a well-defined orbit $s$ of $W$ in the set of pairs $(w, r)$, and $s$ is the desired discrete invariant of $u$. Clearly $s$ depends only on the $G(F)$-conjugacy class of $u$. Turning this around, for a given orbit $s$, we let $\mathfrak{g}(F)_{s}$ denote the subset of $\mathfrak{g}(F)$ consisting of all integral regular semisimple $u$ for which the associated invariant is equal to $s$. 
Observe that the invariant $\left(\delta_{u}, c_{u}\right)$ can be expressed very simply in terms of the $W$-orbit of $(w, r)$. Indeed, we have

$$
\begin{aligned}
\delta_{u}=\delta_{r} & :=\sum_{\alpha \in R} r(\alpha), \\
c_{u}=c_{w} & :=\operatorname{dim} \mathfrak{t}-\operatorname{dim} \mathfrak{t}^{w},
\end{aligned}
$$

$\mathfrak{t}$ being the Lie algebra of $T$, and $\mathfrak{t}^{w}$ denoting the fixed points of $w$ on $\mathfrak{t}$. Therefore the dimension of $X^{u}$ is constant along each subset $\mathfrak{g}(F)_{s}$.

We expect that something much stronger is true, namely that the cohomology of $X^{u}$ is locally constant, in a suitable sense, along each subset $\mathfrak{g}(F)_{s}$. In any case, this is true when the function $r$ is constant (the equivalued case), as can be seen using the Hessenberg pavings of [GKM06].

Thus it is natural to study the subsets $\mathfrak{g}(F)_{s}$. This is best done using the adjoint quotient $\mathbb{A}:=\mathfrak{t} / W$ and the natural morphism

$$
\mathfrak{g}(F) \rightarrow \mathbb{A}(F) .
$$

The set $\mathfrak{g}(F)_{s}$ is the preimage of a subset of $\mathbb{A}(\mathcal{O})$ that we will denote by $\mathbb{A}(\mathcal{O})_{s}$. Given $(w, r)$ in the orbit $s$, we often write $\mathbb{A}(\mathcal{O})_{(w, r)}$ rather than $\mathbb{A}(\mathcal{O})_{s}$.

It is instructive to look at the case when $G=S L_{2}$. Then $\mathbb{A}(F)=F$, and the map (1.0.1) is

$$
\operatorname{det}: \mathfrak{s l}_{2}(F) \rightarrow F \text {. }
$$

Each non-empty subset $\mathbb{A}(\mathcal{O})_{s}$ is of the form

$$
Y_{m}=\{c \in \mathbb{A}(\mathcal{O})=\mathcal{O}: \operatorname{val} c=m\}
$$

for some non-negative integer $m$. The pair $(w, r)$ corresponding to $m$ is determined as follows: $w$ is trivial (respectively, non-trivial) if $m$ is even (respectively, odd), and $r$ is the constant function with value $m / 2$.

The subset $Y_{m}$ is admissible, in the sense that it is the preimage of a subset in $\mathcal{O} / \epsilon^{N} \mathcal{O}$ once $N$ is sufficiently large. This allows us to work with $Y_{m}$ just as if it were finite dimensional. In an obvious sense each $Y_{m}$ is (Zariski) locally closed, irreducible, non-singular of codimension $m$ in $\mathbb{A}(\mathcal{O})$.

One goal of this paper is to prove an analogous statement for any connected reductive $G$ over an algebraically closed field $k$ in which the order of the Weyl 
group is invertible. Theorem 8.2.2 says that $\mathbb{A}(\mathcal{O})_{s}$, when non-empty, is admissible, locally closed, irreducible, and non-singular of codimension

$$
d(w, r)+\left(\delta_{r}+c_{w}\right) / 2
$$

in $\mathbb{A}(\mathcal{O})$. Here $\delta_{r}, c_{w}$ are the same integers as before, and $d(w, r)$ is the codimension of $\mathfrak{t}_{w}(\mathcal{O})_{r}$ in $\mathfrak{t}_{w}(\mathcal{O})$, where $\mathfrak{t}_{w}(\mathcal{O})$ is the twist of $\mathfrak{t}$ by $w$, and $\mathfrak{t}_{w}(\mathcal{O})_{r}$ is a certain subset of $\mathfrak{t}_{w}(\mathcal{O})$ that maps onto $\mathbb{A}(\mathcal{O})_{s}$ under

$$
\mathfrak{t}_{w}(\mathcal{O}) \rightarrow \mathbb{A}(\mathcal{O})
$$

The integer $d(w, r)$ is calculated in Proposition 6.0.1(4).

The second goal of the paper is to relate the geometry of $\mathfrak{t}_{w}(\mathcal{O})_{r}$ to that of $\mathbb{A}(\mathcal{O})_{s}$ using the map

$$
\mathfrak{t}_{w}(\mathcal{O})_{r} \rightarrow \mathbb{A}(\mathcal{O})_{s} .
$$

In Theorem 8.2.2 it is shown that $\mathfrak{t}_{w}(\mathcal{O})_{r}$ is smooth (in a suitable sense) over $\mathbb{A}(\mathcal{O})_{s}$. Theorem 9.1.1 gives a precise description of the structure of this morphism. Combined with Proposition 6.0.1, which concerns $\mathfrak{t}_{w}(\mathcal{O})_{r}$, it yields a clear picture of the structure of each individual stratum $\mathbb{A}(\mathcal{O})_{s}$.

However the methods of this paper shed little light on how the strata fit together. We do not know, for example, whether the closure of $\mathbb{A}(\mathcal{O})_{s}$ is a union of strata.

The paper contains some other results as well. We determine when $\mathbb{A}(\mathcal{O})_{(w, r)}$ is non-empty. Since $\mathfrak{t}_{w}(\mathcal{O})_{r}$ maps onto $\mathbb{A}(\mathcal{O})_{(w, r)}$, this is the same as determining when $\mathfrak{t}_{w}(\mathcal{O})_{r}$ is non-empty, and this is done in Proposition 4.8.2.

Now assume that $\mathbb{A}(\mathcal{O})_{(w, r)}$ is non-empty. We show (Corollary 4.8.4) that if $r$ takes values in $\frac{1}{m} \mathbb{Z}$, then $w^{m}=1$. In particular, if $r$ takes values in $\mathbb{Z}$, then $w=1$. We also show (see subsection 4.9) that if the function $r$ is constant, then the conjugacy class of $w$ is determined by $r$. (This is a simple consequence of Springer's results [Spr74] on regular elements in Weyl groups.) We do not know whether to expect that $w$ is always redundant (more precisely, whether the nonemptiness of both $\mathbb{A}(\mathcal{O})_{(w, r)}$ and $\mathbb{A}(\mathcal{O})_{\left(w^{\prime}, r\right)}$ implies that $w$ and $w^{\prime}$ are conjugate under some element of the Weyl group that fixes $r$.)

A substantial part of this work was done in June, 2000 at the Centre Émile Borel, which we would like to thank both for its financial support and the excellent 
working conditions it provided. It is a pleasure to thank M. Sabitova and the referee for numerous helpful comments on this paper.

\section{Basic notation and Definition of $\mathbb{A}(\mathcal{O})^{\prime}$}

2.1. Notation concerning $G$. Let $G$ be a connected reductive group over an algebraically closed field $k$. We choose a maximal torus $T$ in $G$, and write $\mathfrak{t}$ for its Lie algebra. Throughout this article we will assume that the order $|W|$ of the Weyl group $W$ (of $T$ in $G$ ) is invertible in $k$.

We let $R \subset X^{*}(T)$ denote the set of roots of $T$ in $G$. Occasionally we will need to fix a subset $R^{+} \subset R$ of positive roots. The differential of a root $\alpha$ is an element in the dual space $\mathfrak{t}^{*}$ to $\mathfrak{t}$, and we will abuse notation a bit by also writing $\alpha$ for this element of $\mathfrak{t}^{*}$.

2.2. Quotient variety $\mathbb{A}=\mathfrak{t} / W$. We will need the quotient variety $\mathbb{A}:=\mathfrak{t} / W$, as well as the canonical finite morphism

$$
f: \mathfrak{t} \rightarrow \mathbb{A} \text {. }
$$

The notation $\mathbb{A}$ serves as a reminder that $\mathfrak{t} / W$ is non-canonically isomorphic to affine $n$-space $\mathbb{A}^{n}$ with $n=\operatorname{dim}(T)$. Indeed (see [Bou02]) the $k$-algebra of $W$-invariant polynomial functions on $\mathfrak{t}$ is a polynomial algebra on $n$ homogeneous generators $f_{1}, \ldots, f_{n}$, called basic invariants. Choosing basic invariants $f_{1}, \ldots, f_{n}$, we obtain a morphism

$$
\left(f_{1}, \ldots, f_{n}\right): \mathfrak{t} \rightarrow \mathbb{A}^{n}
$$

which induces an isomorphism $\mathfrak{t} / W \cong \mathbb{A}^{n}$ and allows us to view $f$ as $\left(f_{1}, \ldots, f_{n}\right)$. We will denote by $d_{i}$ the degree of the polynomial $f_{i}$.

2.3. Open subsets of regular elements in $\mathfrak{t}$ and $\mathbb{A}$. Inside $\mathfrak{t}$ we have the $W$-invariant affine open subset $\mathfrak{t}_{\text {reg }}$ consisting of those elements $u \in \mathfrak{t}$ such that $\alpha(u) \neq 0$ for all $\alpha \in R$. Since $|W|$ is invertible in $k$, no root vanishes identically on $\mathfrak{t}$, and therefore $\mathfrak{t}_{\text {reg }}$ is non-empty. The quotient $\mathfrak{t}_{\text {reg }} / W$ is a non-empty affine open subset of $\mathbb{A}$ that we will denote by $\mathbb{A}_{\text {reg }}$.

Picking a basis in the vector space $\mathfrak{t}$, we get coordinates $u_{1}, \ldots, u_{n}$ on $\mathfrak{t}$, and the Jacobian

$$
J_{u}:=\operatorname{det}\left(\frac{\partial f_{i}}{\partial u_{j}}\right)
$$


is a polynomial $J$ in $u=\left(u_{1}, \ldots, u_{n}\right)$ which is known (see [Bou02, Ch. V, no. 5.5, Prop. 6]) to have the form

$$
J_{u}=c \prod_{\alpha \in R^{+}} \alpha(u)
$$

for some non-zero scalar $c \in k$. In particular $\mathfrak{t}_{\text {reg }}$ is the set where the Jacobian does not vanish, and therefore the restriction $f_{\text {reg }}: \mathfrak{t}_{\text {reg }} \rightarrow \mathbb{A}_{\text {reg }}$ of $f$ is an étale covering with Galois group $W$.

Later we will need the well-known identity [Bou02]

$$
\left|R^{+}\right|=\sum_{i=1}^{n}\left(d_{i}-1\right),
$$

which can be proved by calculating the degree of the polynomial $J$ in two different ways.

2.4. Definition of $\mathcal{O}$ and $F$. In fact we will mainly be interested in $\mathbb{A}(\mathcal{O})$, where $\mathcal{O}$ denotes the ring $k[[\epsilon]]$ of formal power series. We also need the fraction field $F=k((\epsilon))$ of $\mathcal{O}$.

2.5. Subset $\mathbb{A}(\mathcal{O})^{\prime}$ of $\mathbb{A}(\mathcal{O})$. We put $\mathbb{A}(\mathcal{O})^{\prime}=\mathbb{A}(\mathcal{O}) \cap \mathbb{A}_{\text {reg }}(F)$, the intersection being taken in $\mathbb{A}(F)$. We stress that this subset is considerably bigger than $\mathbb{A}_{\text {reg }}(\mathcal{O})$. For example, when $G$ is $S L_{2}$, we have $\mathbb{A}(F)=F, \mathbb{A}(\mathcal{O})=\mathcal{O}, \mathbb{A}_{\text {reg }}(F)=$ $F^{\times}, \mathbb{A}_{\text {reg }}(\mathcal{O})=\mathcal{O}^{\times}, \mathbb{A}(\mathcal{O})^{\prime}=\mathcal{O} \backslash\{0\}$. Our first task in this paper is to partition the set $\mathbb{A}(\mathcal{O})^{\prime}$. Roughly speaking, this involves two ingredients: valuations of roots and Weyl group elements. We begin by discussing valuations of roots.

\section{VAluations of Roots: SPlit CASE}

3.1. Normalization of the valuation on $F$. We normalize the valuation on $F$ so that $\operatorname{val}(\epsilon)=1$.

3.2. Definition of $\mathfrak{t}(\mathcal{O})^{\prime}$. Put $\mathfrak{t}(\mathcal{O})^{\prime}:=\mathfrak{t}(\mathcal{O}) \cap \mathfrak{t}_{\text {reg }}(F)$. 
3.3. Definition of $r_{u}$. For any $u \in \mathfrak{t}(\mathcal{O})^{\prime}$ we define a function $r_{u}$ on $R$ by

$$
r_{u}(\alpha)=\operatorname{val} \alpha(u)
$$

for each root $\alpha$. It is clear that $r_{u}$ takes values in the set of non-negative integers.

Since $W$ acts on $R$, it acts on functions $r$ on $R$ by the rule $(w r)(\alpha)=r\left(w^{-1} \alpha\right)$. It is clear that

$$
r_{w u}=w r_{u}
$$

for all $w \in W$ and $u \in \mathfrak{t}(\mathcal{O})^{\prime}$.

3.4. Properties of the function $r_{u}$. Let $u \in \mathfrak{t}(\mathcal{O})^{\prime}$. It is obvious that

$$
r_{u}(-\alpha)=r_{u}(\alpha)
$$

However the non-archimedean property of valuations gives much more than this, as we will now see.

Fix some function $r$ on $R$ with values in the set of non-negative integers. We define a subset $\mathfrak{t}(\mathcal{O})_{r}$ of $\mathfrak{t}(\mathcal{O})^{\prime}$ by

$$
\mathfrak{t}(\mathcal{O})_{r}:=\left\{u \in \mathfrak{t}(\mathcal{O})^{\prime}: r_{u}=r\right\} .
$$

We also use $r$ to define a chain

$$
R=R_{0} \supset R_{1} \supset R_{2} \supset R_{3} \supset \ldots
$$

of subsets

$$
R_{m}:=\{\alpha \in R: r(\alpha) \geq m\} .
$$

We will need the linear subspaces

$$
\mathfrak{a}_{m}:=\left\{u \in \mathfrak{t}: \alpha(u)=0 \quad \forall \alpha \in R_{m}\right\} .
$$

These form an increasing chain

$$
\mathfrak{a}_{0} \subset \mathfrak{a}_{1} \subset \mathfrak{a}_{2} \subset \ldots
$$

with $\mathfrak{a}_{m}=\mathfrak{t}$ for large enough $m$. Finally, for each $m \geq 1$ we will need the subset

$$
\mathfrak{a}_{m}^{\sharp}:=\left\{u \in \mathfrak{a}_{m}: \alpha(u) \neq 0 \quad \forall \alpha \in R_{m-1} \backslash R_{m}\right\}
$$

of $\mathfrak{a}_{m}$. 
Proposition 3.4.1. The set $\mathfrak{t}(\mathcal{O})_{r}$ is non-empty if and only if each subset $R_{m}$ is $\mathbb{Q}$-closed, in the sense that if $\alpha \in R$ is a $\mathbb{Q}$-linear combination of elements in $R_{m}$, then $\alpha$ itself lies in $R_{m}$. Moreover $\mathfrak{t}(\mathcal{O})_{r}$ has the following description: $u \in \mathfrak{t}(\mathcal{O})$ lies in $\mathfrak{t}(\mathcal{O})_{r}$ if and only if the coefficients $u_{j}$ in the power series expansion of $u$ satisfy $u_{j} \in \mathfrak{a}_{j+1}^{\sharp}$ for all $j \geq 0$.

Proof. $(\Longrightarrow)$ Choose $u \in \mathfrak{t}(\mathcal{O})_{r}$ and expand it as a formal power series

$$
u=\sum_{j=0}^{\infty} u_{j} \epsilon^{j}
$$

with coefficients $u_{j} \in \mathfrak{t}$. Clearly $R_{m}=\left\{\alpha \in R: \alpha\left(u_{i}\right)=0 \quad \forall i=0, \ldots, m-1\right\}$. It now follows from Proposition 14.1.1 that $R_{m}$ is $\mathbb{Q}$-closed.

$(\Longleftarrow)$ Assuming that each $R_{m}$ is $\mathbb{Q}$-closed, we must show that $\mathfrak{t}(\mathcal{O})_{r}$ is nonempty. It is clear from the definitions that an element $u \in \mathfrak{t}(\mathcal{O})$ lies in $\mathfrak{t}(\mathcal{O})_{r}$ if and only if the coefficients $u_{j}$ in its power series expansion satisfy $u_{j} \in \mathfrak{a}_{j+1}^{\sharp}$. Thus we just need to show that each $\mathfrak{a}_{j+1}^{\sharp}$ is non-empty. Since $R_{j+1}$ is $\mathbb{Q}$-closed, it is the root system $R_{M}$ of some Levi subgroup $M \supset T$ (see the proof of Proposition 14.1.1(3)). Lemma 14.2.1 then tells us that no root in $R_{j} \backslash R_{j+1}$ vanishes identically on $\mathfrak{a}_{j+1}$, from which it follows immediately that $\mathfrak{a}_{j+1}^{\sharp}$ is non-empty.

\section{TWisted FORMS $\mathfrak{t}_{w}(\mathcal{O})$ AND STRATA $\mathfrak{t}_{w}(\mathcal{O})_{r}$}

The subsets $\mathfrak{t}(\mathcal{O})_{r}$ will help us to understand $\mathbb{A}(\mathcal{O})^{\prime}$, but they are not enough, since the canonical map $\mathfrak{t}(\mathcal{O})^{\prime} \rightarrow \mathbb{A}(\mathcal{O})^{\prime}$ is by no means surjective. In order to get a handle on all elements of $\mathbb{A}(\mathcal{O})^{\prime}$ we need some twisted forms $\mathfrak{t}_{w}(\mathcal{O})$ of $\mathfrak{t}$ over $\mathcal{O}$.

For example, when $G$ is $S L_{2}$ (so that 2 is required to be invertible in $k$ ), the map in question is - up to multiplication by a scalar in $k^{\times}$- the squaring map from $\mathcal{O} \backslash\{0\}$ to $\mathcal{O} \backslash\{0\}$, whose image consists precisely of those elements in $\mathcal{O}$ with even valuation. To obtain the missing elements we need to replace $\mathfrak{t}(\mathcal{O})=\mathcal{O}$ by the $\mathcal{O}$-module of elements in $k\left[\left[\epsilon^{1 / 2}\right]\right]$ having trace 0 in $k[[\epsilon]]$, or, in other words, the $\mathcal{O}$-module (free of rank 1) $\mathcal{O} \epsilon^{1 / 2}$. The squares of the non-zero elements in $\mathcal{O} \epsilon^{1 / 2}$ then yield all elements in $\mathcal{O}$ having odd valuation. The $\mathcal{O}$-module $\mathcal{O} \epsilon^{1 / 2}$ will turn out to be the twisted form $\mathfrak{t}_{w}(\mathcal{O})$ obtained from the non-trivial element $w \in W$. 
We begin by reviewing tamely ramified extensions of $F$. Next we define $\mathfrak{t}_{w}(\mathcal{O})$. Then we use valuations of roots to define subsets $\mathfrak{t}_{w}(\mathcal{O})_{r}$ of $\mathfrak{t}_{w}(\mathcal{O})$. Finally we determine when the strata $\mathfrak{t}_{w}(\mathcal{O})_{r}$ are non-empty.

4.1. Review of $F_{\text {tame }}$. We now need to choose an algebraic closure $\bar{F}$ of $F$. We denote by $F_{\text {sep }}$ the separable closure of $F$ in $\bar{F}$, and by $F_{\text {tame }}$ the maximal tamely ramified extension of $F$ in $F_{\text {sep }}$.

It is well-known that $F_{\text {tame }}$ has the following concrete description. For any positive integer $l$ that is invertible in $k$, we choose an $l$-th $\operatorname{root} \epsilon^{1 / l}$ of $\epsilon$ in $\bar{F}$, and we do this in such a way that $\left(\epsilon^{1 / l m}\right)^{m}=\epsilon^{1 / l}$ for any two positive integers $l, m$ that are both invertible in $k$. The field $F_{l}:=F\left(\epsilon^{1 / l}\right)=k\left(\left(\epsilon^{1 / l}\right)\right)$ is cyclic of degree $l$ over $F$, and is independent of the choice of $l$-th root of $\epsilon$. Moreover $F_{\text {tame }}$ is the union of all the subfields $F_{l}$.

For any positive integer $l$ that is invertible in $k$, we also choose a primitive $l$-th root $\zeta_{l}$ of 1 in $k$, and we do this in such a way that $\left(\zeta_{l m}\right)^{m}=\zeta_{l}$ for any two positive integers $l, m$ that are both invertible in $k$. We use $\zeta_{l}$ to obtain a generator $\tau_{l}$ of $\operatorname{Gal}\left(F_{l} / F\right)$, namely the unique automorphism of $F_{l} / F$ taking $\epsilon^{1 / l}$ to $\zeta_{l} \epsilon^{1 / l}$. These generators are consistent with each other as $l$ varies, and therefore fit together to give an automorphism $\tau_{\infty}$ of $F_{\text {tame }} / F$ whose restriction to each $F_{l}$ is $\tau_{l}$. Clearly $\tau_{\infty}$ is a topological generator of the topologically cyclic group $\operatorname{Gal}\left(F_{\text {tame }} / F\right)$.

4.2. Definition of $\mathfrak{t}_{w}(\mathcal{O})$. Now we can construct the twisted forms of $\mathfrak{t}$ alluded to before. To get such a twist we need to start with an element $w \in W$. We then take $l$ to be the order $o(w)$ of $w$, a positive integer that is invertible in $k$. We write $E$ instead of $F_{l}$ and $\tau_{E}$ instead of $\tau_{l}$. Moreover we write $\epsilon_{E}$ for $\epsilon^{1 / l}$, so that $E=k\left(\left(\epsilon_{E}\right)\right)$ and the valuation $\operatorname{ring} \mathcal{O}_{E}$ in $E$ is $k\left[\left[\epsilon_{E}\right]\right]$.

Then we put

$$
\mathfrak{t}_{w}(\mathcal{O}):=\left\{u \in \mathfrak{t}\left(\mathcal{O}_{E}\right): w \tau_{E}(u)=u\right\} .
$$

More generally, for any $\mathcal{O}$-algebra $A$, we put

$$
\mathfrak{t}_{w}(A):=\mathfrak{t}_{w}(\mathcal{O}) \otimes_{\mathcal{O}} A .
$$

Since it will become clear in subsection 4.3 that $\mathfrak{t}_{w}(\mathcal{O})$ is a free $\mathcal{O}$-module of rank $n$, where $n=\operatorname{dim}_{k} \mathfrak{t}$, we see that $\mathfrak{t}_{w}$ is a scheme over $\mathcal{O}$ isomorphic to affine $n$-space over $\mathcal{O}$. 
Note that only the conjugacy class of $w$ in $W$ really matters: given $x \in W$ we obtain an isomorphism $u \mapsto x u$ from $\mathfrak{t}_{w}(\mathcal{O})$ to $\mathfrak{t}_{x w x^{-1}}(\mathcal{O})$. This shows too that the centralizer $W_{w}\left(\right.$ of $w$ in $W$ ) acts on $\mathfrak{t}_{w}(\mathcal{O})$ (and hence on $\mathfrak{t}_{w}$ over $\mathcal{O}$ ).

4.3. Description of $\mathfrak{t}_{w}(\mathcal{O})$. It is easy to describe $\mathfrak{t}_{w}(\mathcal{O})$ in terms of the eigenspaces for the action of $w$ on $\mathfrak{t}$. Since $w$ has order $l$, the only possible eigenvalues are $l$-th roots of unity. Because $l$ is invertible in $k$, we then have

$$
\mathfrak{t}=\bigoplus_{j=0}^{l-1} \mathfrak{t}(w, j)
$$

where $\mathfrak{t}(w, j)$ denotes the eigenspace $\mathfrak{t}(w, j):=\left\{v \in \mathfrak{t}: w v=\zeta_{l}^{-j} v\right\}$.

An element $u \in \mathfrak{t}\left(\mathcal{O}_{E}\right)$ can be expanded as a formal power series

$$
\sum_{j=0}^{\infty} u_{j} \epsilon_{E}^{j}
$$

with $u_{j} \in \mathfrak{t}$, and we see from (4.2.1) that $u \in \mathfrak{t}_{w}(\mathcal{O})$ if and only if $u_{j} \in \mathfrak{t}(w, j)$ for all $j \geq 0$. Thus there is a canonical $\mathcal{O}$-module isomorphism

$$
\mathfrak{t}_{w}(\mathcal{O}) \cong \bigoplus_{j=0}^{l-1} \mathcal{O} \epsilon_{E}^{j} \otimes_{k} \mathfrak{t}(w, j) .
$$

4.4. Description of $\mathfrak{t}_{w}$ as a fixed point scheme. We write $R_{\mathcal{O}_{E} / \mathcal{O}^{\mathfrak{t}}}$ for the scheme over $\mathcal{O}$ obtained by starting with $\mathfrak{t}$, then extending scalars from $k$ to $\mathcal{O}_{E}$, then (Weil) restricting scalars from $\mathcal{O}_{E}$ to $\mathcal{O}$. For any $\mathcal{O}$-algebra $A$ we then have

$$
\left(R_{\mathcal{O}_{E} / \mathcal{O}}\right)(A)=\mathfrak{t}\left(A \otimes_{\mathcal{O}} \mathcal{O}_{E}\right) .
$$

Of course $R_{\mathcal{O}_{E} / \mathcal{O}^{t}}$ is non-canonically isomorphic to affine space of dimension $n l$ over $\mathcal{O}$.

The automorphism $\tau_{E}$ of $\mathcal{O}_{E} / \mathcal{O}$ induces an automorphism

$$
\tau_{E}: R_{\mathcal{O}_{E} / \mathcal{O}^{\mathfrak{t}}} \rightarrow R_{\mathcal{O}_{E} / \mathcal{O}^{\mathfrak{t}}}
$$

(given on $A$-valued points by the map induced by the $\mathcal{O}$-algebra automorphism $\operatorname{id}_{A} \otimes \tau_{E}$ of $\left.A \otimes_{\mathcal{O}} \mathcal{O}_{E}\right)$. Moreover our $W$-action on $\mathfrak{t}$ induces a $W$-action on $R_{\mathcal{O}_{E} / \mathcal{O}}$ t. The actions of $W$ and $\tau_{E}$ commute, and therefore the cyclic group $\mathbb{Z} / l \mathbb{Z}$ acts on $R_{\mathcal{O}_{E} / \mathcal{O}^{\mathfrak{t}}}$ with the standard generator of that cyclic group acting by $w \circ \tau_{E}$. 
Using (4.3.1), one sees easily that for any $\mathcal{O}$-algebra $A$ we have

$$
\mathfrak{t}_{w}(A)=\left\{u \in \mathfrak{t}\left(A \otimes_{\mathcal{O}} \mathcal{O}_{E}\right): w \tau_{E}(u)=u\right\},
$$

and hence that $\mathfrak{t}_{w}$ is the fixed point scheme (see appendix 15) of the action of $\mathbb{Z} / l \mathbb{Z}$ on $R_{\mathcal{O}_{E} / \mathcal{O}^{t}}$. As a special case of $(4.4 .1)$ we have

$$
\mathfrak{t}_{w}(F)=\left\{u \in \mathfrak{t}(E): w \tau_{E}(u)=u\right\} .
$$

4.5. Definition of $\mathfrak{t}_{w}(\mathcal{O})^{\prime}$. We put

$$
\mathfrak{t}_{w}(\mathcal{O})^{\prime}:=\mathfrak{t}\left(\mathcal{O}_{E}\right)^{\prime} \cap \mathfrak{t}_{w}(\mathcal{O}) .
$$

Thus $u \in \mathfrak{t}_{w}(\mathcal{O})$ lies in $\mathfrak{t}_{w}(\mathcal{O})^{\prime}$ if and only if $\alpha(u) \neq 0$ for all $\alpha \in R$.

4.6. Definition of strata $\mathfrak{t}_{w}(\mathcal{O})_{r}$ in $\mathfrak{t}_{w}(\mathcal{O})^{\prime}$. We extend the valuation on the field $F$ to a valuation, still denoted val, on $\bar{F}$. In particular we have $\operatorname{val}\left(\epsilon^{1 / l}\right)=$ $1 / l$.

Let $\mathcal{R}$ denote the set of functions on $R$ with values in the set of non-negative rational numbers. For $r \in \mathcal{R}$ we put

$$
\mathfrak{t}_{w}(\mathcal{O})_{r}:=\left\{u \in \mathfrak{t}_{w}(\mathcal{O}): \operatorname{val} \alpha(u)=r(\alpha) \quad \forall \alpha \in R\right\} .
$$

It is clear that $\mathfrak{t}_{w}(\mathcal{O})^{\prime}$ is the disjoint union of the strata $\mathfrak{t}_{w}(\mathcal{O})_{r}$, many of which are empty.

The Weyl group acts on itself by conjugation, and it also acts on $\mathcal{R}$ (see subsection 3.3); thus we have an action of $W$ on the set of pairs $(w, r) \in W \times \mathcal{R}$. Note that only the $W$-orbit of $(w, r)$ really matters: given $x \in W$ we obtain an isomorphism $u \mapsto x u$ from $\mathfrak{t}_{w}(\mathcal{O})_{r}$ to $\mathfrak{t}_{x w x^{-1}}(\mathcal{O})_{x r}$.

4.7. Freeness of the $W_{w}$-action on $\mathfrak{t}_{w}\left(\mathcal{O} / \epsilon^{N} \mathcal{O}\right)_{r<N}$. The centralizer $W_{w}$ acts freely on $\mathfrak{t}_{w}(\mathcal{O})^{\prime}$ by Proposition 14.1.1. Now let $N$ be a positive integer. We are going to define an open subset $\mathfrak{t}_{w}\left(\mathcal{O} / \epsilon^{N} \mathcal{O}\right)_{r<N}$ of the $k$-variety $\mathfrak{t}_{w}\left(\mathcal{O} / \epsilon^{N} \mathcal{O}\right.$ ) (see 16.1) on which $W_{w}$ acts freely. Here is the definition:

$$
\mathfrak{t}_{w}\left(\mathcal{O} / \epsilon^{N} \mathcal{O}\right)_{r<N}:=\left\{u \in \mathfrak{t}_{w}\left(\mathcal{O} / \epsilon^{N} \mathcal{O}\right): \alpha(u) \neq 0 \quad \forall \alpha \in R\right\} .
$$

(Note that $\alpha(u)$ is an element of the ring $\mathcal{O}_{E} / \epsilon^{N} \mathcal{O}_{E}$.) The set $\mathfrak{t}_{w}\left(\mathcal{O} / \epsilon^{N} \mathcal{O}\right)_{r<N}$ can also be described as the image in $\mathfrak{t}_{w}\left(\mathcal{O} / \epsilon^{N} \mathcal{O}\right)$ of all strata $\mathfrak{t}_{w}(\mathcal{O})_{r}$ for which $r$ satisfies the condition $r(\alpha)<N$ for all $\alpha \in R$. 
Now we verify that $W_{w}$ acts freely on $\mathfrak{t}_{w}\left(\mathcal{O} / \epsilon^{N} \mathcal{O}\right)_{r<N}$. Let $u \in \mathfrak{t}_{w}\left(\mathcal{O} / \epsilon^{N} \mathcal{O}\right)_{r<N}$ and expand it as

$$
u=\sum_{j=0}^{N l-1} u_{j} \epsilon_{E}^{j} .
$$

Suppose that some element $x \in W_{w}$ fixes $u$. Then $x$ fixes each coefficient $u_{j}$. It follows from Proposition 14.1.1 (1) that $x$ lies in the Weyl group of the root system consisting of all roots $\alpha \in R$ such that $\alpha\left(u_{j}\right)=0$ for all $j$. Since $\alpha(u) \neq 0$ for all $\alpha \in R$, there are no such roots, and therefore $x=1$.

4.8. Which strata are non-empty? We are now going to determine which strata $\mathfrak{t}_{w}(\mathcal{O})_{r}$ are non-empty. (Only the $W$-orbit of $(w, r)$ matters.) We begin by listing some useful necessary conditions.

Let $u \in \mathfrak{t}_{w}(\mathcal{O})^{\prime}$. Then $\alpha(u) \in \mathcal{O}_{E}$ and thus val $\alpha(u) \in \frac{1}{l} \mathbb{Z}$. Therefore a necessary condition for non-emptiness of $\mathfrak{t}_{w}(\mathcal{O})_{r}$ is that $r$ take values in $\frac{1}{l} \mathbb{Z}$. Of course this statement can be sharpened a little, since a particular root $\alpha$ may be defined over $k\left(\left(\epsilon^{1 / l^{\prime}}\right)\right)$ for some divisor $l^{\prime}$ of $l$, in which case it is necessary that $r(\alpha)$ lie in $\frac{1}{l^{\prime}} \mathbb{Z}$.

Now assume that $r$ does take values in $\frac{1}{l} \mathbb{Z}$, and define an integer valued function $r_{E}$ on $R$ by $r_{E}(\alpha)=\operatorname{lr}(\alpha)$. It is clear from the definitions that

$$
\mathfrak{t}_{w}(\mathcal{O})_{r}=\mathfrak{t}\left(\mathcal{O}_{E}\right)_{r_{E}} \cap \mathfrak{t}_{w}(\mathcal{O}) .
$$

(Here we are applying definitions we have already made for $F$ to the field $E$, so that when interpreting the right side of this equality one should be thinking of the normalized valuation on $E$, rather than the one that extends the valuation on $F$. That is why we need $r_{E}$ instead of $r$.) Now Proposition 3.4.1 tells us exactly when $\mathfrak{t}\left(\mathcal{O}_{E}\right)_{r_{E}}$ is non-empty. We conclude that another necessary condition for the non-emptiness of $\mathfrak{t}_{w}(\mathcal{O})_{r}$ is that the subset

$$
R_{m}:=\{\alpha \in R: r(\alpha) \geq m / l\}
$$

of $R$ be $\mathbb{Q}$-closed for every non-negative integer $m$. We now assume that this condition on $r$ also holds.

Our stratum $\mathfrak{t}_{w}(\mathcal{O})_{r}$ might still be empty. To settle the question we need once again to consider the vector spaces

$$
\mathfrak{a}_{m}:=\left\{u \in \mathfrak{t}: \alpha(u)=0 \quad \forall \alpha \in R_{m}\right\}
$$


and their open subsets

$$
\mathfrak{a}_{m}^{\sharp}:=\left\{u \in \mathfrak{a}_{m}: \alpha(u) \neq 0 \quad \forall \alpha \in R_{m-1} \backslash R_{m}\right\} .
$$

Lemma 4.8.1. Let $u \in \mathfrak{t}\left(\mathcal{O}_{E}\right)$, and expand $u$ as a power series

$$
\sum_{j=0}^{\infty} u_{j} \epsilon_{E}^{j}
$$

with $u_{j} \in \mathfrak{t}$. Then $u \in \mathfrak{t}_{w}(\mathcal{O})_{r}$ if and only if $u_{j} \in \mathfrak{t}(w, j) \cap \mathfrak{a}_{j+1}^{\sharp}$ for all $j \geq 0$. Consequently $\mathfrak{t}_{w}(\mathcal{O})_{r}$ is non-empty if and only if $\mathfrak{t}(w, j) \cap \mathfrak{a}_{j+1}^{\sharp}$ is non-empty for all $j \geq 0$.

Proof. We observed in 4.3 that $u \in \mathfrak{t}_{w}(\mathcal{O})$ if and only if $u_{j} \in \mathfrak{t}(w, j)$ for all $j \geq 0$. Moreover it follows from Proposition 3.4.1 that $u \in \mathfrak{t}\left(\mathcal{O}_{E}\right)_{r_{E}}$ if and only if $u_{j} \in \mathfrak{a}_{j+1}^{\sharp}$ for all $j \geq 0$.

We can reformulate the non-emptiness result in the last lemma in a slightly better way, but for this we first need to note that there is another obvious necessary condition for non-emptiness. Indeed, for any $u \in \mathfrak{t}\left(\mathcal{O}_{E}\right)^{\prime}$ we have $r_{\tau_{E}(u)}=r_{u}$ (obvious) and hence $r_{w \tau_{E}(u)}=w r_{u}$ (use (3.3.1)). It follows that if $u \in \mathfrak{t}_{w}(\mathcal{O})^{\prime}$, then $r_{u}=w r_{u}$.

Thus we see that if $\mathfrak{t}_{w}(\mathcal{O})_{r}$ is non-empty, then $w$ stabilizes $r$, from which it follows that $w$ stabilizes the subsets $R_{m}$ and the subspaces $\mathfrak{a}_{j}$ of $\mathfrak{t}$, so that we obtain an action of $w$ on each quotient space $\mathfrak{a}_{j+1} / \mathfrak{a}_{j}$. In this situation we may consider the eigenspace

$$
\left(\mathfrak{a}_{j+1} / \mathfrak{a}_{j}\right)(w, j):=\left\{v \in \mathfrak{a}_{j+1} / \mathfrak{a}_{j}: w v=\zeta_{l}^{-j} v\right\} .
$$

Let us also note that since each root in $R_{j}$ vanishes identically on $\mathfrak{a}_{j}$, our subset $\mathfrak{a}_{j+1}^{\sharp}$ is the preimage under $\mathfrak{a}_{j+1} \rightarrow \mathfrak{a}_{j+1} / \mathfrak{a}_{j}$ of the set

$$
\left(\mathfrak{a}_{j+1} / \mathfrak{a}_{j}\right)^{\sharp}:=\left\{u \in \mathfrak{a}_{j+1} / \mathfrak{a}_{j}: \alpha(u) \neq 0 \quad \forall \alpha \in R_{j} \backslash R_{j+1}\right\} .
$$

Proposition 4.8.2. The stratum $\mathfrak{t}_{w}(\mathcal{O})_{r}$ is non-empty if and only if the following four conditions hold.

(1) $r$ takes values in $\frac{1}{l} \mathbb{Z}$.

(2) $R_{m}$ is $\mathbb{Q}$-closed for all $m \geq 0$.

(3) $w r=r$.

(4) $\left(\mathfrak{a}_{j+1} / \mathfrak{a}_{j}\right)(w, j) \cap\left(\mathfrak{a}_{j+1} / \mathfrak{a}_{j}\right)^{\sharp}$ is non-empty for all $j \geq 0$. 
Note that the fourth condition makes sense only when the third condition holds.

Proof. This follows from the previous lemma, since $\mathfrak{t}(w, j) \cap \mathfrak{a}_{j+1}$ projects onto $\left(\mathfrak{a}_{j+1} / \mathfrak{a}_{j}\right)(w, j)$. Indeed, when $w r=r$, the canonical surjection $\mathfrak{a}_{j+1} \rightarrow \mathfrak{a}_{j+1} / \mathfrak{a}_{j}$ is equivariant for the action of $w$, and therefore the $\zeta_{l}^{-j}$-eigenspace of $w$ on $\mathfrak{a}_{j+1}$ maps onto the $\zeta_{l}^{-j}$-eigenspace of $w$ on $\mathfrak{a}_{j+1} / \mathfrak{a}_{j}$. Here we used that the order $l$ of $w$ is invertible in $k$.

Proposition 4.8.3. Suppose that $\mathfrak{t}_{w}(\mathcal{O})_{r}$ is non-empty, and suppose that $r(\alpha) \in$ $\mathbb{Z}$ for all $\alpha \in R$. Then $w=1$.

Proof. Let $u \in \mathfrak{t}_{w}(\mathcal{O})_{r}$ and expand $u$ as a power series

$$
\sum_{j=0}^{\infty} u_{j} \epsilon_{E}^{j} .
$$

We are going to apply Proposition 14.1 .1 to the subset $S:=\left\{u_{m l}: m=\right.$ $0,1,2, \ldots\}$. Since $u_{m l} \in \mathfrak{t}(w, m l)$, we see that $w$ fixes each element of $S$ and therefore lies in the subgroup $W_{S}=W\left(R_{S}\right)$ of Proposition 14.1.1. However $R_{S}$ is empty, since for any $\alpha \in R$ we have $\alpha\left(u_{r(\alpha) l}\right) \neq 0$. Therefore $w=1$.

Corollary 4.8.4. Let $m$ be a positive integer. Suppose that $\mathfrak{t}_{w}(\mathcal{O})_{r}$ is non-empty, and suppose that $r(\alpha) \in \frac{1}{m} \mathbb{Z}$ for all $\alpha \in R$. Then $w^{m}=1$.

Proof. The idea is to extend scalars from $F$ to $F_{m}$. We denote the valuation ring in $F_{m}$ by $\mathcal{O}_{m}$. Then we have

$$
\mathfrak{t}_{w}(\mathcal{O})_{r} \subset \mathfrak{t}_{w^{m}}\left(\mathcal{O}_{m}\right)_{m r},
$$

which shows that $\mathfrak{t}_{w^{m}}\left(\mathcal{O}_{m}\right)_{m r}$ is non-empty. Since $m r$ takes integral values, the previous result, applied to $F_{m}$ rather than $F$, tells us that $w^{m}=1$.

4.9. Equivalued strata. We say that a stratum $\mathfrak{t}_{w}(\mathcal{O})_{r}$ is equivalued if the function $r$ is constant. We will now use Proposition 4.8.2 to reduce the problem of classifying non-empty equivalued strata to a problem that has already been solved by Springer [Spr74]. Because Springer works over a base-field of characteristic 0, we temporarily do so too, just in this subsection. (It seems quite likely that our usual hypothesis that $|W|$ be invertible in $k$ suffices, but we have not checked this carefully.) 
We need to recall the following definition (due to Springer): an element $w \in W$ is said to be regular if there exists a non-zero eigenvector $u$ of $w$ in $\mathfrak{t}$ that is regular (in the sense that no root $\alpha$ vanishes on $u$ ). When $w$ is regular of order $l$, Springer [Spr74] shows that the eigenspace $\mathfrak{t}(w, j)$ contains a regular element of $\mathfrak{t}$ if and only if $j$ is relatively prime to $l$.

Proposition 4.9.1. Let $a / b$ be a non-negative rational number written in least common terms, so that $b$ is positive and $(a, b)=1$. Let $r$ be the constant function on $R$ with value $a / b$, and let $w \in W$. Then $\mathfrak{t}_{w}(\mathcal{O})_{r}$ is non-empty if and only if $w$ is regular of order $b$.

Proof. We use our usual notation. In particular $l$ denotes the order of $w$. From Proposition 4.8.2 we see that $\mathfrak{t}_{w}(\mathcal{O})_{r}$ is non-empty if and only if $b$ divides $l$ and the eigenspace $\mathfrak{t}(w, a l / b)$ contains a regular element of $\mathfrak{t}$. The proposition follows from this and the result of Springer mentioned just before the statement of the proposition.

Let us now recall a beautiful result from Springer's paper (see [Spr74, Theorem 4.2], as well as the remarks following the proof of that theorem): all regular elements in $W$ of a given order are conjugate. Combining this with the previous proposition, we see that for a given $a / b$ in least common terms (letting $r$ denote, as before, the constant function with value $a / b$ ), there are two possibilities. The first is that there is no regular element of $W$ having order $b$. In this case there are no non-empty strata $\mathfrak{t}_{w}(\mathcal{O})_{r}$. The second is that there are regular elements in $W$ having order $b$, in which case there is a single conjugacy class of such elements, and $\mathfrak{t}_{w}(\mathcal{O})_{r}$ is non-empty if and only if $w$ lies in this conjugacy class. The somewhat surprising conclusion is that $w$ is essentially redundant: given $a / b$, there is at most one $W$-orbit of pairs $(w, r)$ for which $r$ is the constant function with value $a / b$ and $\mathfrak{t}_{w}(\mathcal{O})_{r}$ is non-empty.

This raises an obvious question. Let $r$ be a non-negative rational valued function on $R$, and let $W_{r}$ denote the stabilizer of $r$ in $W$. Suppose that $\mathfrak{t}_{w}(\mathcal{O})_{r}$ and $\mathfrak{t}_{w^{\prime}}(\mathcal{O})_{r}$ are both non-empty. Is it then true that $w, w^{\prime}$ are conjugate under $W_{r}$ ? This is a question, not a conjecture. We do not know whether to expect a positive or negative answer.

We should also remark that Springer [Spr74] gives a list of the regular elements in the Weyl group of each irreducible root system. Together with the proposition 
we just proved, this gives a classification of all non-empty equivalued strata. Take $G$ to be $S L(n)$, for example. Then we get non-empty equivalued strata from pairs $(w, a / b)$ (with $a / b$ in least common terms and $b=o(w)$ ) for which $w$ is a power of either an $n$-cycle or an $(n-1)$-cycle.

\section{Admissible subsets of $X(\mathcal{O})$}

Before we describe the structure of the sets $\mathfrak{t}_{w}(\mathcal{O})_{r}$ we need a few preliminary remarks and definitions. Consider a scheme $X$ of finite type over $\mathcal{O}$. Then for any positive integer $N$ Greenberg's functor [Gre61] (see appendix 16 for a review) provides us with a scheme of finite type over $k$ whose set of $k$-points is $X\left(\mathcal{O} / \epsilon^{N} \mathcal{O}\right)$. In general the natural $k$-morphism $X\left(\mathcal{O} / \epsilon^{N+1} \mathcal{O}\right) \rightarrow X\left(\mathcal{O} / \epsilon^{N} \mathcal{O}\right)$ can be complicated, but when $X$ is smooth over $\mathcal{O}$, as we will always assume in this section, Greenberg [Gre63] shows that $X\left(\mathcal{O} / \epsilon^{N+1} \mathcal{O}\right)$ is an affine space bundle over $X\left(\mathcal{O} / \epsilon^{N} \mathcal{O}\right)$ (more precisely, a torsor under the pullback to $X\left(\mathcal{O} / \epsilon^{N} \mathcal{O}\right.$ ) of the tangent bundle on $X(k))$. In particular each $X\left(\mathcal{O} / \epsilon^{N} \mathcal{O}\right)$ is then smooth over $k$, and the mapping $X\left(\mathcal{O} / \epsilon^{N+1} \mathcal{O}\right) \rightarrow X\left(\mathcal{O} / \epsilon^{N} \mathcal{O}\right)$ is open and surjective.

In case $X$ is affine $n$-space $\mathbb{A}^{n}$ over $\mathcal{O}$ (for example, $\mathfrak{t}_{w}(\mathcal{O})$ or $\mathbb{A}(\mathcal{O})$, the two main cases of interest in this paper), the situation is particularly simple, since then $X\left(\mathcal{O} / \epsilon^{N} \mathcal{O}\right)$ is $\mathbb{A}_{k}^{n N}$, and $X\left(\mathcal{O} / \epsilon^{N+1} \mathcal{O}\right) \rightarrow X\left(\mathcal{O} / \epsilon^{N} \mathcal{O}\right)$ is a projection map $\mathbb{A}_{k}^{n(N+1)} \rightarrow \mathbb{A}_{k}^{n N}$.

5.1. Admissible subsets. For any positive integer $N$ we write $p_{N}: X(\mathcal{O}) \rightarrow$ $X\left(\mathcal{O} / \epsilon^{N} \mathcal{O}\right)$ for the canonical surjection (induced of course by $\mathcal{O} \rightarrow \mathcal{O} / \epsilon^{N} \mathcal{O}$ ). We say that a subset $Y$ of $X(\mathcal{O})$ is $N$-admissible if $Y=p_{N}^{-1} p_{N} Y$, in which case we introduce $Y_{N}$ as a convenient notation for $p_{N} Y$. If $Y$ is $N$-admissible, it is clear that $Y$ is $N^{\prime}$-admissible for all $N^{\prime} \geq N$. We say that $Y$ is admissible if there exists $N$ such that $Y$ is $N$-admissible.

5.2. Topological notions for admissible subsets. Let $Y$ be an admissible subset of $X(\mathcal{O})$. We say that $Y$ is open (respectively, closed, locally closed, irreducible) in $X(\mathcal{O})$ if $Y_{N}$ is open (respectively, closed, locally closed, irreducible) in $X\left(\mathcal{O} / \epsilon^{N} \mathcal{O}\right)$ for some (equivalently, every) positive integer $N$ such that $Y$ is $N$-admissible. (To see the equivalence of "some" and "every" use Lemma 13.1.2.) 
We define the closure $\bar{Y}$ of $Y$ in $X(\mathcal{O})$ as follows: choose $N$ such that $Y$ is $N$ admissible and put $\bar{Y}=p_{N}^{-1} \bar{Y}_{N}$, where $\bar{Y}_{N}$ of course denotes the closure of $Y_{N}$ in $X\left(\mathcal{O} / \epsilon^{N} \mathcal{O}\right)$; by Lemma 13.1.1(3) $\bar{Y}$ is independent of the choice of $N$.

5.3. Non-singularity for admissible subsets. Now assume that $Y$ is a locally closed admissible subset of $X(\mathcal{O})$. For $N$ such that $Y$ is $N$-admissible we regard $Y_{N}$ as a reduced scheme of finite type over $k$ by putting the induced reduced subscheme structure on the locally closed subset $Y_{N} \subset X\left(\mathcal{O} / \epsilon^{N} \mathcal{O}\right)$. We say that $Y$ is non-singular if $Y_{N}$ is non-singular for some (equivalently, every) positive integer $N$ such that $Y$ is $N$-admissible. (To see the equivalence of "some" and "every" use Lemma 13.2.1.)

Now assume that $Y$ is indeed non-singular, and let $y \in Y$. We want to define the tangent space $T_{Y, y}$ to $Y$ at $y$. This will be an admissible $k$-linear subspace of the tangent space $T_{X(\mathcal{O}), y}$ (see 16.2) to $X(\mathcal{O})$ at $y$.

To define $T_{Y, y}$ we choose $N$ so large that $Y$ is $N$-admissible, and we denote by $\bar{y}$ the image of $y$ in $Y_{N}$. Then the tangent space $T_{Y_{N}, \bar{y}}$ is a linear subspace of the tangent space $T_{X\left(\mathcal{O} / \epsilon^{N} \mathcal{O}\right), \bar{y}}$. Recall from 16.2 that there is a canonical surjection

$$
T_{X(\mathcal{O}), y} \rightarrow T_{X\left(\mathcal{O} / \epsilon^{N} \mathcal{O}\right), \bar{y}}
$$

which identifies $T_{X\left(\mathcal{O} / \epsilon^{N} \mathcal{O}\right), \bar{y}}$ with $T_{X(\mathcal{O}), y} \otimes_{\mathcal{O}}\left(\mathcal{O} / \epsilon^{N} \mathcal{O}\right)$. We now define $T_{Y, y}$ to be the inverse image under (5.3.1) of $T_{Y_{N}, \bar{y}}$. It is easy to see that $T_{Y, y}$ is independent of the choice of $N$.

5.4. Smoothness for maps between admissible subsets. Now let $f: X \rightarrow$ $X^{\prime}$ be an $\mathcal{O}$-morphism between smooth schemes $X, X^{\prime}$ over $\mathcal{O}$. For each positive integer $N$ Greenberg's functor yields a $k$-morphism

$$
f_{N}: X\left(\mathcal{O} / \epsilon^{N} \mathcal{O}\right) \rightarrow X^{\prime}\left(\mathcal{O} / \epsilon^{N} \mathcal{O}\right)
$$

Suppose that $Y, Y^{\prime}$ are admissible locally closed subsets of $X(\mathcal{O}), X^{\prime}(\mathcal{O})$ respectively, with the property that $f(Y) \subset Y^{\prime}$, and let $g: Y \rightarrow Y^{\prime}$ denote the map obtained by restriction from $f: X(\mathcal{O}) \rightarrow X^{\prime}(\mathcal{O})$. For each $N$ such that both $Y, Y^{\prime}$ are $N$-admissible, we obtain (by restriction from $f_{N}$ ) a $k$-morphism

$$
g_{N}: Y_{N} \rightarrow Y_{N}^{\prime}
$$

As usual we put the induced reduced subscheme structures on $Y_{N}, Y_{N}^{\prime}$. 
For $M \geq N$ there is a commutative square

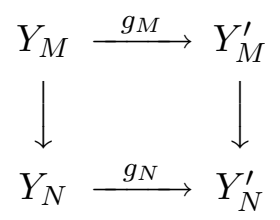

in which the vertical arrows (the obvious surjections) are smooth (by Lemma 13.2.1). It then follows from EGA IV (17.11.1) that if $g_{M}$ is smooth, then $g_{N}$ is also smooth. However, if $g_{N}$ is smooth, it is not necessarily the case that $g_{M}$ is smooth.

We say that $Y$ is smooth over $Y^{\prime}$ (or that $g: Y \rightarrow Y^{\prime}$ is smooth) if $g_{M}$ is smooth for all $M \geq N$. The remarks we just made show that this condition is independent of the choice of $N$ for which $Y, Y^{\prime}$ are both $N$-admissible.

It is evident from the definitions that if $g: Y \rightarrow Y^{\prime}$ is smooth, and $Y^{\prime}$ is non-singular, then $Y$ is non-singular. Using Lemma 13.2.1 one checks easily that if $Y$ is smooth over $Y^{\prime}$, then $g^{-1} Z^{\prime}$ is smooth over $Z^{\prime}$ for any admissible locally closed subset $Z^{\prime}$ of $X^{\prime}(\mathcal{O})$ such that $Z^{\prime} \subset Y^{\prime}$.

Lemma 5.4.1. Now assume that both $Y, Y^{\prime}$ are non-singular. Define the differential $d g_{y}$ of $g: Y \rightarrow Y^{\prime}$ at $y \in Y$ to be the k-linear map $d g_{y}: T_{Y, y} \rightarrow T_{Y^{\prime}, f(y)}$ obtained by restricting the differential $d f_{y}: T_{X(\mathcal{O}), y} \rightarrow T_{X^{\prime}(\mathcal{O}), f(y)}$ to the tangent space $T_{Y, y}$. Then $Y$ is smooth over $Y^{\prime}$ if and only if the differential

$$
d g_{y}: T_{Y, y} \rightarrow T_{Y^{\prime}, f(y)}
$$

is surjective for all $y \in Y$.

Proof. Let $y \in Y$ and put $y^{\prime}:=f(y)$. For any integer $M$ with $M \geq N$ we denote by $y_{M}$ the image of $y$ under the canonical surjection $Y \rightarrow Y_{M}$. We do the same for $y^{\prime}$, so that $y_{M}^{\prime}=g_{M}\left(y_{M}\right)$. We then have a commutative square

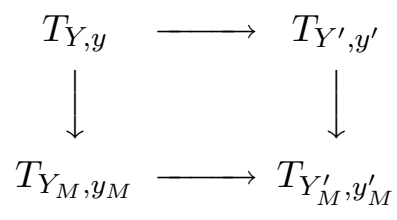

in which the horizontal maps are differentials and the vertical maps are the canonical surjections. 
The implication $(\Longleftarrow)$ of the lemma is now clear, since (5.4.2) together with the surjectivity of (5.4.1) shows that each $g_{M}$ is a submersion.

It remains to prove the reverse implication $(\Longrightarrow)$. To simplify notation we put $L:=T_{X(\mathcal{O}), y}\left(\right.$ a free $\mathcal{O}$-module of finite rank) and $V:=T_{Y, y}($ an $N$-admissible $k$ linear subspace of $L$ ), and we use parallel notation for $Y^{\prime}$. Then (5.4.2) becomes the square

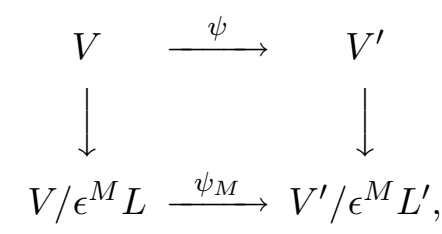

where $\psi$ is the $k$-linear map obtained by restriction from the $\mathcal{O}$-linear map $\varphi$ : $L \rightarrow L^{\prime}$ defined by $\varphi:=d f_{y}$.

Our assumption that $Y$ is smooth over $Y^{\prime}$ tells us that $\psi_{M}$ is surjective for all $M \geq N$, which just means that

$$
\varphi V+\epsilon^{M} L^{\prime}=V^{\prime}
$$

for all $M \geq N$. Since $V^{\prime} \supset \epsilon^{N} L^{\prime}$, we conclude that

$$
\epsilon^{N} L^{\prime} \subset \bigcap_{M \geq N}\left(\varphi L+\epsilon^{M} L^{\prime}\right)=\varphi L .
$$

(Here we used that $\varphi L$ is an $\mathcal{O}$-submodule of $L^{\prime}$.) Since $V \supset \epsilon^{N} L$, we see that

$$
\varphi V \supset \epsilon^{N} \varphi L \supset \epsilon^{2 N} L^{\prime} .
$$

Taking $M=2 N$ in (5.4.3), we conclude that $\varphi V=V^{\prime}$, showing that (5.4.1) is surjective, as desired.

Corollary 5.4.2. Suppose that $Y$ is smooth over $Y^{\prime}$. Then for each $y \in Y$ the F-linear map obtained by extension of scalars from the differential

$$
d f_{y}: T_{X(\mathcal{O}), y} \rightarrow T_{X^{\prime}(\mathcal{O}), f(y)}
$$

is surjective. In other words, $Y$ is necessarily contained in the subset of $X(F)$ consisting of all points at which the F-morphism obtained by extension of scalars from $f: X \rightarrow X^{\prime}$ is smooth.

Proof. Put $y^{\prime}:=f(y)$. Choose $N$ for which both $Y, Y^{\prime}$ are $N$-admissible. Write $y_{N}^{\prime}$ for the image of $y^{\prime}$ under the canonical surjection $Y^{\prime} \rightarrow Y_{N}^{\prime}$, and let $Z^{\prime}$ denote the preimage of $y_{N}^{\prime}$ in $Y^{\prime}$. We have noted before that $g^{-1} Z^{\prime}$ is smooth over $Z^{\prime}$. 
Since $Z^{\prime}$ is obviously non-singular, so too is $g^{-1} Z^{\prime}$. The previous lemma, applied to $g^{-1} Z^{\prime} \rightarrow Z^{\prime}$, then tells us that the map

$$
T_{g^{-1} Z^{\prime}, y} \rightarrow T_{Z^{\prime}, y^{\prime}}
$$

is surjective, and hence that the image of the map (5.4.4) contains $\epsilon^{N} T_{X^{\prime}(\mathcal{O}), y^{\prime}}$. Therefore the $F$-linear map obtained from (5.4.4) is surjective.

5.5. Codimensions of admissible subsets. Now suppose that $X(k)$ is irreducible (which implies that $X\left(\mathcal{O} / \epsilon^{N} \mathcal{O}\right)$ is irreducible for every positive integer $N$ ). When $Y$ is an admissible, locally closed, irreducible subset of $X(\mathcal{O})$, we define its codimension in $X(\mathcal{O})$ to be the codimension of $Y_{N}$ in $X\left(\mathcal{O} / \epsilon^{N} \mathcal{O}\right)$ for any $N$ such that $Y$ is $N$-admissible; it is easy to see that this notion of codimension is independent of the choice of $N$.

\section{Structure of the Strata $\mathfrak{t}_{w}(\mathcal{O})_{r}$}

We now continue the discussion of $\mathfrak{t}_{w}(\mathcal{O})_{r}$, retaining the notation used before. In particular $l$ denotes the order of $w$, and $\mathfrak{a}_{m}$ denotes the linear subspace defined in subsection 4.8. We now have the right vocabulary to discuss the structure of $\mathfrak{t}_{w}(\mathcal{O})_{r}$.

In the next result we will need the stabilizer $W_{w, r}$ of the pair $(w, r)$; thus $W_{w, r}$ consists of elements $x \in W$ such that $x w=w x$ and $x r=r$. When $w$ stabilizes $r$ (and hence the subspaces $\mathfrak{a}_{0} \subset \mathfrak{a}_{1} \subset \mathfrak{a}_{2} \subset \cdots$ of $\mathfrak{t}$ ), we may then consider the eigenspaces

$$
\left(\mathfrak{t} / \mathfrak{a}_{j+1}\right)(w, j):=\left\{v \in\left(\mathfrak{t} / \mathfrak{a}_{j+1}\right): w v=\zeta_{l}^{-j} v\right\} .
$$

Proposition 6.0.1. Assume that $Y:=\mathfrak{t}_{w}(\mathcal{O})_{r}$ is non-empty, which guarantees in particular that $r$ takes values in $\frac{1}{l} \mathbb{Z}$ and that $w$ stabilizes $r$. Let $N$ be a positive integer large enough that $r(\alpha)<N$ for all $\alpha \in R$. Then the following conclusions hold.

(1) The subset $Y$ of $\mathfrak{t}_{w}(\mathcal{O})$ is $N$-admissible. Thus $Y$ is the preimage of its image $Y_{N}$ in $\mathfrak{t}_{w}\left(\mathcal{O} / \epsilon^{N} \mathcal{O}\right)$.

(2) The closure $\bar{Y}$ of $Y$ in $\mathfrak{t}_{w}(\mathcal{O})$ is the admissible $k$-linear subspace

$$
\left\{u \in \mathfrak{t}_{w}(\mathcal{O}): \operatorname{val} \alpha(u) \geq r(\alpha) \quad \forall \alpha \in R\right\}
$$

of $\mathfrak{t}_{w}(\mathcal{O})$. 
(3) The subset $Y \subset \bar{Y}$ is the complement of finitely many admissible $k$-linear hyperplanes $H_{1}, \ldots, H_{m}$ in $\bar{Y}$. Consequently $Y$ is locally closed, irreducible, and non-singular. The group $W_{w, r}$ preserves $Y, \bar{Y}$ and permutes the hyperplanes $H_{1}, \ldots, H_{m}$ in $\bar{Y}$; moreover, $W_{w, r}$ acts freely on $Y_{N}$.

(4) The codimension of $Y$ in $\mathfrak{t}_{w}(\mathcal{O})$ is the same as that of the linear subspace $\bar{Y}$, namely

$$
\operatorname{dim}_{k}\left(\mathfrak{t}_{w}(\mathcal{O}) / \bar{Y}\right)=\sum_{j=0}^{\infty} \operatorname{dim}_{k}\left(\mathfrak{t} / \mathfrak{a}_{j+1}\right)(w, j) .
$$

When $w=1$, this expression for the codimension simplifies to

$$
\sum_{j=0}^{\infty} j \cdot \operatorname{dim}_{k}\left(\mathfrak{a}_{j+1} / \mathfrak{a}_{j}\right) .
$$

Proof. (1) Suppose that $u \in \mathfrak{t}_{w}(\mathcal{O})_{r}$ and that $u^{\prime} \in \epsilon^{N} \mathfrak{t}_{w}(\mathcal{O})$. For each root $\alpha$ we must show that $\alpha\left(u+u^{\prime}\right)$ has the same valuation as $\alpha(u)$. This is clear from our hypothesis that $r(\alpha)<N$.

(2) By Lemma 4.8.1 the non-emptiness of $\mathfrak{t}_{w}(\mathcal{O})_{r}$ implies the non-emptiness of $\mathfrak{t}(w, j) \cap \mathfrak{a}_{j+1}^{\sharp}$ for all $j \geq 0$. Since $\mathfrak{t}(w, j) \cap \mathfrak{a}_{j+1}^{\sharp}$ is the complement of finitely many hyperplanes in $\mathfrak{t}(w, j) \cap \mathfrak{a}_{j+1}$, we see that the closure of $\mathfrak{t}(w, j) \cap \mathfrak{a}_{j+1}^{\sharp}$ is $\mathfrak{t}(w, j) \cap \mathfrak{a}_{j+1}$. Lemma 4.8.1 then implies that the closure of $\mathfrak{t}_{w}(\mathcal{O})_{r}$ is the set of all $u=\sum_{j=0}^{\infty} u_{j} \epsilon_{E}^{j} \in \mathfrak{t}_{w}(\mathcal{O})$ such that $u_{j} \in \mathfrak{a}_{j+1}$ for all $j \geq 0$. On the other hand $u$ lies in

$$
\left\{u \in \mathfrak{t}_{w}(\mathcal{O}): \operatorname{val} \alpha(u) \geq r(\alpha) \quad \forall \alpha \in R\right\}
$$

if and only if $\alpha\left(u_{j}\right)=0$ whenever $j / l<r(\alpha)$, and this happens if and only if $u_{j} \in \mathfrak{a}_{j+1}$ (by the very definition of $\mathfrak{a}_{j+1}$ ).

(3) The proof of (2) shows that $Y$ is the complement of finitely many admissible hyperplanes in $\bar{Y}$. The freeness of the action of $W_{w, r}$ on $Y_{N}$ follows from the freeness (see 4.7) of the action of $W_{w}$ on the larger set $\mathfrak{t}_{w}\left(\mathcal{O} / \epsilon^{N} \mathcal{O}\right)_{r<N}$. The remaining statements are clear.

(4) The description of $\bar{Y}$ given in (2) shows that

$$
\operatorname{dim}_{k}\left(\mathfrak{t}_{w}(\mathcal{O}) / \bar{Y}\right)=\sum_{j=0}^{\infty} \operatorname{dim}_{k} \mathfrak{t}(w, j) /\left(\mathfrak{t}(w, j) \cap \mathfrak{a}_{j+1}\right) .
$$


Since the order of $w$ is invertible in $k$, we see that $\mathfrak{t}(w, j) /\left(\mathfrak{t}(w, j) \cap \mathfrak{a}_{j+1}\right)$ can be identified with $\left(\mathfrak{t} / \mathfrak{a}_{j+1}\right)(w, j)$. (In other words, the image under $\mathfrak{t} \rightarrow \mathfrak{t} / \mathfrak{a}_{j+1}$ of the $\zeta_{l}^{-j}$-eigenspace of $w$ on $\mathfrak{t}$ is the $\zeta_{l}^{-j}$-eigenspace of $w$ on $\mathfrak{t} / \mathfrak{a}_{j+1}$.)

Finally, when $w=1$, we have $\left(\mathfrak{t} / \mathfrak{a}_{j+1}\right)(w, j)=\mathfrak{t} / \mathfrak{a}_{j+1}$, whose dimension is $\sum_{j^{\prime}=j+1}^{\infty} \operatorname{dim}_{k}\left(\mathfrak{a}_{j^{\prime}+1} / \mathfrak{a}_{j^{\prime}}\right)$. This proves the last statement in (4).

\section{Strata in $\mathbb{A}(\mathcal{O})^{\prime}$}

We now stratify $\mathbb{A}(\mathcal{O})^{\prime}$. We obtain the desired strata in $\mathbb{A}(\mathcal{O})^{\prime}$ as images of the strata $\mathfrak{t}_{w}(\mathcal{O})_{r}$ that we have already studied.

7.1. Definition of the map $f_{w}: \mathfrak{t}_{w}(\mathcal{O}) \rightarrow \mathbb{A}(\mathcal{O})$. Let $w \in W$. The map $\mathfrak{t}\left(\mathcal{O}_{E}\right) \rightarrow \mathbb{A}\left(\mathcal{O}_{E}\right)$ on $\mathcal{O}_{E}$-points induced by our morphism $f$ restricts to a map

$$
f_{w}: \mathfrak{t}_{w}(\mathcal{O}) \rightarrow \mathbb{A}(\mathcal{O})
$$

Recall that the centralizer $W_{w}$ (of $w$ in $W$ ) acts on $\mathfrak{t}_{w}(\mathcal{O})$. This action preserves the fibers of the map $f_{w}$.

In fact $f_{w}$ comes from a morphism of schemes over $\mathcal{O}$ that will also be denoted simply by $f_{w}: \mathfrak{t}_{w} \rightarrow \mathbb{A}_{\mathcal{O}}$, with $\mathbb{A}_{\mathcal{O}}$ denoting the $\mathcal{O}$-scheme obtained from $\mathbb{A}$ by extending scalars from $k$ to $\mathcal{O}$. This is best understood using the point of view (see 4.4) that $\mathfrak{t}_{w}$ is the fixed point scheme of a $\mathbb{Z} / \mathbb{Z}$-action on $R_{\mathcal{O}_{E} / \mathcal{O}} \mathfrak{t}$. (We again remind the reader that fixed point schemes are discussed in appendix 15.)

By $R_{\mathcal{O}_{E} / \mathcal{O}} \mathbb{A}$ we will of course mean the scheme obtained from $\mathbb{A}$ by extending scalars from $k$ to $\mathcal{O}_{E}$, and then (Weil) restricting scalars from $\mathcal{O}_{E}$ to $\mathcal{O}$. Thus

$$
\left(R_{\mathcal{O}_{E} / \mathcal{O}} \mathbb{A}\right)(A)=\mathbb{A}\left(A \otimes_{\mathcal{O}} \mathcal{O}_{E}\right)
$$

for any $\mathcal{O}$-algebra $A$.

As in subsection 4.4 the automorphism $\tau_{E}$ of $\mathcal{O}_{E} / \mathcal{O}$ induces an automorphism $\tau_{E}$ of $R_{\mathcal{O}_{E} / \mathcal{O}} \mathbb{A}$ of order $l$, so that we obtain an action of $\mathbb{Z} / l \mathbb{Z}$ on $R_{\mathcal{O}_{E} / \mathcal{O}} \mathbb{A}$. The fixed point scheme of $\mathbb{Z} / l \mathbb{Z}$ on $R_{\mathcal{O}_{E} / \mathcal{O}} \mathbb{A}$ is $\mathbb{A}_{\mathcal{O}}$, as one sees from the (easy) fact that $A$ is the set of fixed points of $\operatorname{id}_{A} \otimes \tau_{E}$ on $A \otimes_{\mathcal{O}} \mathcal{O}_{E}$ for any $\mathcal{O}$-algebra (or even $\mathcal{O}$-module) $A$. 
Starting from $f: \mathfrak{t} \rightarrow \mathbb{A}$, then extending scalars to $\mathcal{O}_{E}$, then restricting scalars to $\mathcal{O}$, we get an $\mathcal{O}$-morphism

$$
R(f): R_{\mathcal{O}_{E} / \mathcal{O}^{\mathfrak{t}}} \rightarrow R_{\mathcal{O}_{E} / \mathcal{O}} \mathbb{A}
$$

which intertwines the automorphism $w \tau_{E}$ of $R_{\mathcal{O}_{E} / \mathcal{O}^{\mathfrak{t}}}$ with the automorphism $\tau_{E}$ of $R_{\mathcal{O}_{E} / \mathcal{O}} \mathbb{A}$, and hence induces the desired $\mathcal{O}$-morphism $f_{w}$ upon taking fixed points under $\mathbb{Z} / l \mathbb{Z}$.

7.2. Fibers of $\mathfrak{t}_{w}(\mathcal{O})^{\prime} \rightarrow \mathbb{A}(\mathcal{O})^{\prime}$. Recall that the centralizer $W_{w}$ acts on $\mathfrak{t}_{w}(\mathcal{O})$, preserving the fibers of $f_{w}$. We claim that $W_{w}$ acts simply transitively on every non-empty fiber of the restriction of $f_{w}$ to $\mathfrak{t}_{w}(\mathcal{O})^{\prime}$. Indeed, let $u, u^{\prime} \in \mathfrak{t}_{w}(\mathcal{O})^{\prime}$ and suppose that $f_{w}(u)=f_{w}\left(u^{\prime}\right)$. Then there exists unique $x \in W$ such that $x u=u^{\prime}$. Using that $u, u^{\prime}$ are fixed by $w \tau_{E}$, we see that

$$
w x \tau_{E}(u)=u^{\prime}=x w \tau_{E}(u)
$$

and hence that $w x=x w$, as claimed.

7.3. Definition of the strata $\mathbb{A}(\mathcal{O})_{s}$ in $\mathbb{A}(\mathcal{O})^{\prime}$. Consider a pair $(w, r) \in W \times \mathcal{R}$. We denote by $\mathbb{A}(\mathcal{O})_{w, r}$ the image of $\mathfrak{t}_{w}(\mathcal{O})_{r}$ under the map $f_{w}$. It is clear that $\mathbb{A}(\mathcal{O})_{w, r}$ depends only on the $W$-orbit of $(w, r)$ (with, as usual, $W$ acting on itself by conjugation). Thus it is often better to index the strata by the set $\mathcal{S}$ of orbits of $W$ on $W \times \mathcal{R}$. In other words, given $s \in \mathcal{S}$, represented by a pair $(w, r)$, we will often write $\mathbb{A}(\mathcal{O})_{s}$ instead of $\mathbb{A}(\mathcal{O})_{w, r}$.

Since $\mathbb{A}(\mathcal{O})_{s}$ is by definition obtained as the image of $\mathfrak{t}_{w}(\mathcal{O})_{r}$, Proposition 4.8.2 tells us when $\mathbb{A}(\mathcal{O})_{s}$ is non-empty.

Lemma 7.3.1. The set $\mathbb{A}(\mathcal{O})^{\prime}$ is the disjoint union of the strata $\mathbb{A}(\mathcal{O})_{s}$.

Proof. Let $c \in \mathbb{A}(\mathcal{O})^{\prime}$. Consider the fiber over $c$ of the map $\mathfrak{t}(\bar{F}) \rightarrow \mathbb{A}(\bar{F})$ induced by our morphism $f: \mathfrak{t} \rightarrow \mathbb{A}$. (Recall that for any $k$-algebra $A$ we have $\mathfrak{t}(A)=$ $\mathfrak{t} \otimes_{k} A$.) The Weyl group $W$ acts simply transitively on this fiber. Moreover, since $f_{\text {reg }}$ is étale, the fiber is actually contained in the subset $\mathfrak{t}\left(F_{\mathrm{sep}}\right)$ of $\mathfrak{t}(\bar{F})$. The action of $\operatorname{Gal}\left(F_{\text {sep }} / F\right)$ on $\mathfrak{t}\left(F_{\text {sep }}\right)$ preserves the fiber because $c$ is defined over $F$ (and even over $\mathcal{O}$ ).

Now choose an element $u$ in the fiber. For any element $\tau \in \operatorname{Gal}\left(F_{\mathrm{sep}} / F\right)$ there exists a unique $w_{\tau} \in W$ such that $w_{\tau} \tau(u)=u$, and $\tau \mapsto w_{\tau}$ is a homomorphism from $\operatorname{Gal}\left(F_{\text {sep }} / F\right)$ to $W$. Since $|W|$ is invertible in $k$, this homomorphism factors 
through the quotient $\operatorname{Gal}\left(F_{\text {tame }} / F\right)$ of $\operatorname{Gal}\left(F_{\text {sep }} / F\right)$, and in fact we will now simply regard $\tau \mapsto w_{\tau}$ as a homomorphism from $\operatorname{Gal}\left(F_{\text {tame }} / F\right)$ to $W$. Recall from before the topological generator $\tau_{\infty}$ of $\operatorname{Gal}\left(F_{\text {tame }} / F\right)$. Putting $w:=w_{\tau_{\infty}}$, we have associated an element $w \in W$ to the element $u$ in the fiber. As usual we write $l$ for $o(w)$ and $E$ for $F_{l}$.

It is clear from the definitions that $u \in \mathfrak{t}_{w}(F)$ and that $u \mapsto c$ under our morphism $f$. The valuative criterion of properness, applied to the proper morphism $f$ and the valuation ring $\mathcal{O}_{E}$, implies that $u \in \mathfrak{t}\left(\mathcal{O}_{E}\right)$ and hence that $u \in \mathfrak{t}_{w}(\mathcal{O})$. Define $r \in \mathcal{R}$ by $r(\alpha):=\operatorname{val} \alpha(u)$. Then $u$ lies in the stratum $\mathfrak{t}_{w}(\mathcal{O})_{r}$, and therefore $c$ lies in the stratum $\mathbb{A}(\mathcal{O})_{s}$. Thus we have shown that our strata exhaust $\mathbb{A}(\mathcal{O})$.

It remains to establish disjointness of our strata. Suppose that $u_{1} \in \mathfrak{t}_{w_{1}}(\mathcal{O})_{r_{1}}$ and that $u_{2} \in \mathfrak{t}_{w_{2}}(\mathcal{O})_{r_{2}}$. Suppose further that $u_{1}$ and $u_{2}$ have the same image $c$ in $\mathbb{A}(\mathcal{O})$. We must show that $\left(w_{1}, r_{1}\right)$ and $\left(w_{2}, r_{2}\right)$ are in the same $W$-orbit. This is easy: there exists a unique element $x \in W$ such that $x u_{1}=u_{2}$, and this element $x$ transforms $\left(w_{1}, r_{1}\right)$ into $\left(w_{2}, r_{2}\right)$.

Lemma 7.3.2. We have

$$
f_{w}^{-1}\left(\mathbb{A}(\mathcal{O})_{s}\right)=\coprod_{x \in W_{w} / W_{w, r}} \mathfrak{t}_{w}(\mathcal{O})_{x r}
$$

where, as usual, $W_{w, r}$ denotes the stabilizer in $W$ of the pair $(w, r)$, and $s$ denotes the $W$-orbit of $(w, r)$. Moreover, $W_{w, r}$ acts simply transitively on each fiber of $\mathfrak{t}_{w}(\mathcal{O})_{r} \rightarrow \mathbb{A}(\mathcal{O})_{s}$.

Proof. This follows from the discussion in subsection 7.2 and the obvious equality (valid for any $x \in W_{w}$ )

$$
\mathfrak{t}_{w}(\mathcal{O})_{x r}=x \mathfrak{t}_{w}(\mathcal{O})_{r}
$$

8. Structure of the strata $\mathbb{A}(\mathcal{O})_{s}$ In $\mathbb{A}(\mathcal{O})^{\prime}$

In this section, after introducing a couple of definitions, we are going to formulate Theorem 8.2.2, which describes the structure of the strata $\mathbb{A}(\mathcal{O})_{s}$ in $\mathbb{A}(\mathcal{O})^{\prime}$. 
8.1. Definitions of $\delta_{r}$ and $c_{w}$. Let $s \in \mathcal{S}$ be the $W$-orbit of the pair $(w, r)$. Let us assume that the stratum $\mathbb{A}(\mathcal{O})_{s}$ is non-empty (equivalently: $\mathfrak{t}_{w}(\mathcal{O})_{r}$ is non-empty). As usual we put $l:=o(w)$ and $E:=F_{l}$.

So far we have not used the $F$-torus $T_{w}$ that goes along with $\mathfrak{t}_{w}(F)$. This torus splits over $E$ and is obtained by using $w$ to twist the (split) torus over $F$ obtained by extension of scalars from $T$. In particular we have

$$
T_{w}(F)=\left\{t \in T(E): w \tau_{E}(t)=t\right\},
$$

and the Lie algebra of $T_{w}$ is canonically isomorphic to $\mathfrak{t}_{w}(F)$.

There is a canonical $G(F)$-conjugacy class of $F$-embeddings $T_{w} \rightarrow G$ (with image a maximal $F$-torus in $G$ ). This is well-known (perhaps see [GKM06] for a rather concrete presentation of this material). Fixing such an embedding, we may identify $\mathfrak{t}_{w}(F)$ with a Cartan subalgebra in $\mathfrak{g}(F)$. For any regular element $u \in \mathfrak{t}_{w}(F)$ the centralizer in $\mathfrak{g}(F)$ of $u$ is equal to $\mathfrak{t}_{w}(F)$, and we have the usual non-zero scalar $\Delta(u)$ in $F$ defined by

$$
\Delta(u):=\operatorname{det}\left(\operatorname{ad}(u) ; \mathfrak{g}(F) / \mathfrak{t}_{w}(F)\right) .
$$

Clearly this determinant is simply the product of the values on $u$ of all the roots of our Cartan subalgebra. Therefore, if $u \in \mathfrak{t}_{w}(\mathcal{O})_{r}$, we have

$$
\operatorname{val} \Delta(u)=\delta_{r},
$$

where

$$
\delta_{r}:=\sum_{\alpha \in R} r(\alpha) .
$$

Note that $\delta_{r}$ is a non-negative integer. (It is clearly non-negative, and our expression for it as the valuation of $\Delta(u) \in F^{\times}$shows that it is an integer. We could have defined $\delta_{r}$ without this digression concerning $T_{w}$, but then it would not have been clear that $\delta_{r}$ is an integer.)

Since $r(-\alpha)=r(\alpha)$ for all $\alpha \in R$ (because of our assumption that $\mathfrak{t}_{w}(\mathcal{O})_{r}$ be non-empty), we also have

$$
\delta_{r}=2 \sum_{\alpha \in R^{+}} r(\alpha)
$$

We need some more notation before we state the next result. We denote by $\mathfrak{t}^{w}$ the set of fixed points of $w$ on $\mathfrak{t}$, and we denote by $c_{w}$ the integer

$$
c_{w}:=\operatorname{dim}_{k} \mathfrak{t}-\operatorname{dim}_{k} \mathfrak{t}^{w} .
$$


Equivalently (because of our hypothesis on the characteristic of our base field), $c_{w}$ is the dimension of $T$ minus the dimension of the maximal $F$-split torus in $T$.

8.2. Valuation of the Jacobian of $f_{w}$. Recall the map $f_{w}: \mathfrak{t}_{w}(\mathcal{O}) \rightarrow \mathbb{A}(\mathcal{O})$, which, as we saw in 7.1, comes from a morphism of schemes over $\mathcal{O}$. Our chosen basic invariants allow us to identify $\mathbb{A}$ with $\mathbb{A}^{n}$, and by choosing an $\mathcal{O}$-basis of the free $\mathcal{O}$-module $\mathfrak{t}_{w}(\mathcal{O})$, we may also identify the $\mathcal{O}$-scheme $\mathfrak{t}_{w}$ with $\mathbb{A}_{\mathcal{O}}^{n}$.

These identifications allow us to think of the differential $\left(d f_{w}\right)_{u}$ of $f_{w}$ at $u \in$ $\mathfrak{t}_{w}(\mathcal{O})$ concretely as a square matrix $D_{u} \in M_{n} \mathcal{O}$, as in 10.1. In the next lemma we will compute the valuation of $\operatorname{det} D_{u}$ for $u \in \mathfrak{t}_{w}(\mathcal{O})_{r}$, as this will be needed in the proof of Theorem 8.2.2. Observe that making a different choice of $\mathcal{O}$-basis for $\mathfrak{t}_{w}(\mathcal{O})$ does not affect the valuation of $\operatorname{det} D_{u}$, so that it makes sense to write $\operatorname{val} \operatorname{det}\left(d f_{w}\right)_{u}$.

Lemma 8.2.1. For any $u \in \mathfrak{t}_{w}(\mathcal{O})_{r}$ the non-negative integer val $\operatorname{det}\left(d f_{w}\right)_{u}$ is equal to $\left(\delta_{r}+c_{w}\right) / 2$.

Proof. We can calculate this determinant after extending scalars from $\mathcal{O}$ to $\mathcal{O}_{E}$. Then we are dealing with the $\mathcal{O}_{E}$-linear map

$$
\operatorname{id}_{E} \otimes\left(d f_{w}\right)_{u}: \mathcal{O}_{E} \otimes_{\mathcal{O}} \mathfrak{t}_{w}(\mathcal{O}) \rightarrow \mathcal{O}_{E}^{n}
$$

which is none other than the restriction of

$$
(d f)_{u}: \mathfrak{t}\left(\mathcal{O}_{E}\right) \rightarrow \mathcal{O}_{E}^{n}
$$

to the subspace $\mathcal{O}_{E} \otimes_{\mathcal{O}} \mathfrak{t}_{w}(\mathcal{O})$ of $\mathfrak{t}\left(\mathcal{O}_{E}\right)$. We conclude that

$$
\operatorname{val} \operatorname{det}\left(d f_{w}\right)_{u}=\operatorname{val} \operatorname{det}(d f)_{u}+\frac{1}{l} \operatorname{dim}_{k} \frac{\mathfrak{t}\left(\mathcal{O}_{E}\right)}{\mathcal{O}_{E} \otimes_{\mathcal{O}} \mathfrak{t}_{w}(\mathcal{O})} \text {. }
$$

In order to prove the lemma it is enough to check that

$$
\operatorname{val} \operatorname{det}(d f)_{u}=\delta_{r} / 2
$$

and that

$$
\operatorname{dim}_{k} \frac{\mathfrak{t}\left(\mathcal{O}_{E}\right)}{\mathcal{O}_{E} \otimes_{\mathcal{O}} \mathfrak{t}_{w}(\mathcal{O})}=l c_{w} / 2 .
$$

Now (8.2.1) follows from (2.3.1), and (8.2.2) is [Bez96, Lemma 3]. (Bezrukavnikov treats simply connected groups over $\mathbb{C}$, but his proof goes through in our situation. For this we just need to show that the representation of $W$ on $\mathfrak{t}$ is isomorphic to 
its own contragredient. Since $|W|$ is invertible in $k$, it is enough to check that the $W$-modules $\mathfrak{t}$ and $\mathfrak{t}^{*}$ have the same character, and this is clear, since $\mathfrak{t}$ is obtained by tensoring $X_{*}(T)$ with $k$, so that all character values lie in the prime field.)

Let us introduce one more bit of notation before stating the next theorem. We put $e(w, r):=\left(\delta_{r}+c_{w}\right) / 2$. It follows from Lemma 8.2.1 that $e(w, r)$ is an integer (non-negative, of course).

Theorem 8.2.2. Let $s \in \mathcal{S}$ be the $W$-orbit of the pair $(w, r)$. Let us assume that the stratum $\mathbb{A}(\mathcal{O})_{s}$ is non-empty. Then we have the following conclusions.

(1) The subset $\mathbb{A}(\mathcal{O})_{\text {s }}$ of $\mathbb{A}(\mathcal{O})$ is admissible; more precisely, it is $N$-admissible whenever $N>2 e(w, r)$. Moreover it is locally closed, irreducible and nonsingular.

(2) The codimension of $\mathbb{A}(\mathcal{O})_{s}$ in $\mathbb{A}(\mathcal{O})$ is given by

$$
d(w, r)+e(w, r),
$$

where $d(w, r)$ is the codimension of $\mathfrak{t}_{w}(\mathcal{O})_{r}$ in $\mathfrak{t}_{w}(\mathcal{O})$.

$(3) \mathfrak{t}_{w}(\mathcal{O})_{r}$ is smooth over $\mathbb{A}(\mathcal{O})_{s}$. Here we are using the notion of smoothness discussed in 5.4 .

Proof. This will be proved in section 11 .

\section{Relation Between the strata $\mathfrak{t}_{w}(\mathcal{O})_{r}$ And $\mathbb{A}(\mathcal{O})_{s}$}

9.1. Set-up for this section. Consider a non-empty stratum $\mathfrak{t}_{w}(\mathcal{O})_{r}$, and let $s$ again denote the $W$-orbit of the pair $(w, r)$. We abbreviate $e(w, r)$ to $e$. We now have a good understanding of the strata $\mathfrak{t}_{w}(\mathcal{O})_{r}$ and $\mathbb{A}(\mathcal{O})_{s}$, but we would like to supplement this by analyzing the smooth morphism

$$
\mathfrak{t}_{w}(\mathcal{O})_{r} \rightarrow \mathbb{A}(\mathcal{O})_{s}
$$

obtained by restriction from $f_{w}$.

To do so we choose $N$ large enough that $N>2 e$, and use the fact that both $\mathfrak{t}_{w}(\mathcal{O})_{r}$ and $\mathbb{A}(\mathcal{O})_{s}$ are $N$-admissible (see Proposition 6.0.1 and Theorem 8.2.2). (For additional details see the first few lines of the proof of Theorem 8.2.2, where it is shown that $\mathfrak{t}_{w}(\mathcal{O})_{r}$ is even $(N-e)$-admissible.) In Theorem 9.1.1 we will gain an understanding of

$$
\mathfrak{t}_{w}(\mathcal{O})_{r} \rightarrow \mathbb{A}(\mathcal{O})_{s}
$$


by analyzing the smooth morphism

$$
\mathfrak{t}_{w}\left(\mathcal{O} / \epsilon^{N} \mathcal{O}\right)_{r} \rightarrow \mathbb{A}\left(\mathcal{O} / \epsilon^{N} \mathcal{O}\right)_{s} .
$$

Here we have written $\mathfrak{t}_{w}\left(\mathcal{O} / \epsilon^{N} \mathcal{O}\right)_{r}$ for the image of $\mathfrak{t}_{w}(\mathcal{O})_{r}$ in $\mathfrak{t}_{w}\left(\mathcal{O} / \epsilon^{N} \mathcal{O}\right)$. Similarly we have written $\mathbb{A}\left(\mathcal{O} / \epsilon^{N} \mathcal{O}\right)_{s}$ for the image of $\mathbb{A}(\mathcal{O})_{s}$ in $\mathbb{A}\left(\mathcal{O} / \epsilon^{N} \mathcal{O}\right)$.

Theorem 9.1.1 makes use of a rank $e$ vector bundle $\tilde{V}$ over $\mathbb{A}\left(\mathcal{O} / \epsilon^{N} \mathcal{O}\right)_{s}$ that will be constructed in the course of proving the theorem. This vector bundle acts on $\mathfrak{t}_{w}\left(\mathcal{O} / \epsilon^{N} \mathcal{O}\right)_{r}$ over $\mathbb{A}\left(\mathcal{O} / \epsilon^{N} \mathcal{O}\right)_{s}$. The group $W_{w, r}$ also acts on $\mathfrak{t}_{w}\left(\mathcal{O} / \epsilon^{N} \mathcal{O}\right)_{r}$ over $\mathbb{A}\left(\mathcal{O} / \epsilon^{N} \mathcal{O}\right)_{s}$. The two actions commute, so the group scheme $H:=W_{w, r} \times \tilde{V}$ (product over $k$ ) over $\mathbb{A}\left(\mathcal{O} / \epsilon^{N} \mathcal{O}\right)_{s}$ also acts on $\mathfrak{t}_{w}\left(\mathcal{O} / \epsilon^{N} \mathcal{O}\right)_{r}$ over $\mathbb{A}\left(\mathcal{O} / \epsilon^{N} \mathcal{O}\right)_{s}$.

Theorem 9.1.1. The space $\mathfrak{t}_{w}\left(\mathcal{O} / \epsilon^{N} \mathcal{O}\right)_{r}$ over $\mathbb{A}\left(\mathcal{O} / \epsilon^{N} \mathcal{O}\right)_{s}$ is a torsor under H. In particular we can factorize the morphism $\mathfrak{t}_{w}\left(\mathcal{O} / \epsilon^{N} \mathcal{O}\right)_{r} \rightarrow \mathbb{A}\left(\mathcal{O} / \epsilon^{N} \mathcal{O}\right)_{s}$ as the composition of two morphisms, one of which is a bundle of affine spaces of dimension e, and the other of which is an étale covering that is Galois with group $W_{w, r}$. The factorization can be done in either order.

Proof. This will be proved in section 12 .

\section{Behavior of admissibility under polynomial maps $f: \mathcal{O}^{n} \rightarrow \mathcal{O}^{n}$}

In this section we will establish some technical results needed for the proofs of the theorems we have stated. It is the morphism $f_{w}: \mathfrak{t}_{w}(\mathcal{O}) \rightarrow \mathbb{A}(\mathcal{O})$ that we need to understand, but it is conceptually simpler to work in the more general context of polynomial maps $f: \mathcal{O}^{n} \rightarrow \mathcal{O}^{n}$. The key Lemma 10.3.1, a generalization of Hensel's lemma, is a variant of a special case of one of the main results of Greenberg's paper [Gre66].

10.1. Set-up for this section. Consider the polynomial ring $A=\mathcal{O}\left[X_{1}, \ldots, X_{n}\right]$. Thus $\operatorname{Spec} A$ is affine $n$-space $\mathbb{A}^{n}$ over $\mathcal{O}$. In this section we study a morphism $\mathbf{f}: \mathbb{A}^{n} \rightarrow \mathbb{A}^{n}$ of schemes over $\mathcal{O}$. Thus $\mathbf{f}$ is given by an $n$-tuple $\mathbf{f}=\left(f_{1}, \ldots, f_{n}\right)$ of elements in $A$.

We write $L$ for $\mathcal{O}^{n}$, the set of $\mathcal{O}$-valued points of $\mathbb{A}^{n}$. We are mainly interested in the map

$$
f: L \rightarrow L
$$

on $\mathcal{O}$-valued points induced by our morphism $\mathbf{f}: \mathbb{A}^{n} \rightarrow \mathbb{A}^{n}$. 
We regard the differential $d \mathbf{f}$ of $\mathbf{f}$ concretely as an element of $M_{n} A$, the ring of square matrices of size $n$ with entries in $A$. Of course the matrix entries are the partial derivatives $\partial f_{j} / \partial X_{i}$. We denote by $D_{x}$ the value of $d \mathbf{f}$ at $x \in L$; thus $D_{x} \in M_{n} \mathcal{O}$ and $D_{x}$ can also be viewed as an $\mathcal{O}$-linear map $D_{x}: L \rightarrow L$. It is evident that the reduction modulo $\epsilon^{N}$ of $D_{x}$ depends only on $x$ modulo $\epsilon^{N}$. (Here $N$ is any non-negative integer.)

We put $g=\operatorname{det}(d \mathbf{f}) \in A$; clearly $g(x)=\operatorname{det} D_{x}$ for $x \in L$. The reduction modulo $\epsilon^{N}$ of $g(x)$ depends only on $x$ modulo $\epsilon^{N}$. For $x \in L$ we write $d(x)$ for the valuation of $g(x)$. Thus $d(x)$ is a non-negative integer when $g(x) \neq 0$, and $d(x)=+\infty$ when $g(x)=0$.

10.2. The linear case. The situation is of course especially simple when our morphism $f$ is linear. In this subsection we suppose that $f: L \rightarrow L$ is given by multiplication by a matrix $A \in M_{n} \mathcal{O}$ whose determinant is non-zero, and we put $d:=\operatorname{val} \operatorname{det} A$. Thus the $\mathcal{O}$-module $L / A L$ has length $d$, hence is killed by $\epsilon^{d}$, which is to say that

$$
\epsilon^{d} L \subset A L .
$$

Lemma 10.2.1. Let $Y$ be a subset of $L$ that is admissible, locally closed, irreducible, non-singular of codimension a in $L$. Then $A Y$ is admissible, locally closed, irreducible, non-singular of codimension $a+d$ in $L$.

Proof. Easy.

For any non-negative integer $N$ we denote by $A_{N}$ the reduction of $A$ modulo $\epsilon^{N}$. We view $A_{N}$ as an $\mathcal{O} / \epsilon^{N} \mathcal{O}$-linear map $A_{N}: L / \epsilon^{N} L \rightarrow L / \epsilon^{N} L$. In the next lemma we will see that for $N \geq d$ the kernel of $A_{N}$ is always $d$-dimensional and is even independent of $N$, up to canonical isomorphism. The kind of canonical isomorphism that will come up is of the following type: for any integers $M, N$ with $M \leq N$, there is a canonical isomorphism $L / \epsilon^{N-M} L \cong \epsilon^{M} L / \epsilon^{N} L$, given of course by multiplication by $\epsilon^{M}$.

Lemma 10.2.2. Suppose that $N \geq d$. Then $\operatorname{ker}\left(A_{N}\right)$ is $d$-dimensional and is contained in the subspace $\epsilon^{N-d} L / \epsilon^{N} L$ of $L / \epsilon^{N} L$. Moreover, under the canonical isomorphism $\epsilon^{N-d} L / \epsilon^{N} L \cong L / \epsilon^{d} L$, the subspace $\operatorname{ker}\left(A_{N}\right)$ goes over to the subspace $\operatorname{ker}\left(A_{d}\right)$. 
Proof. First we note that

$$
\operatorname{dim} \operatorname{ker}\left(A_{N}\right)=\operatorname{dim} \operatorname{cok}\left(A_{N}\right)=\operatorname{dim} L / A L=d .
$$

Here we used (10.2.1) and $N \geq d$ to see that $A L \supset \epsilon^{N} L$.

Since $\operatorname{det}(A) \neq 0$, we may consider the inverse $A^{-1} \in M_{n} F$ of $A$. From (10.2.1) we obtain

$$
A^{-1} \epsilon^{N} L \subset \epsilon^{N-d} L \subset L
$$

Therefore

$$
\operatorname{ker}\left(A_{N}\right)=A^{-1} \epsilon^{N} L / \epsilon^{N} L \cong A^{-1} \epsilon^{d} L / \epsilon^{d} L=\operatorname{ker}\left(A_{d}\right)
$$

and

$$
\operatorname{ker}\left(A_{N}\right)=A^{-1} \epsilon^{N} L / \epsilon^{N} L \subset \epsilon^{N-d} L / \epsilon^{N} L
$$

as desired.

10.3. Solving the equation $f\left(x^{\prime}\right)=y$ by successive approximations. As mentioned before, the next lemma is a variant of results of Greenberg. Since the precise statement we need is not stated explicitly in [Gre66], we thought it best to write out in full our adaptation of Greenberg's arguments.

Lemma 10.3.1. Let $x \in L$ and assume that $g(x) \neq 0$. Let $M$ be an integer such that $M>d(x)$. Then

$$
f\left(x+\epsilon^{M} L\right) \supset f(x)+\epsilon^{M+d(x)} L .
$$

More precisely

$$
f\left(x+\epsilon^{M} L\right)=f(x)+D_{x}\left(\epsilon^{M} L\right) .
$$

Proof. In this proof we abbreviate $d(x)$ to $d$. We begin with two observations. The first is that

$$
\epsilon^{d} L \subset D_{x} L,
$$

an instance of (10.2.1). The second is that

$$
f(x+h) \equiv f(x)+D_{x} h \quad \bmod \epsilon^{2 M} L
$$

for all $h \in \epsilon^{M} L$, as one sees from the Taylor expansion of $f$ about the point $x$. (In fact (10.3.2) is valid for all $x \in L$ and all non-negative $M$; the hypothesis $M>d$ is not needed at this point.) 
It follows from (10.3.1) that $\epsilon^{M+d} L \subset D_{x}\left(\epsilon^{M} L\right)$; therefore the first assertion of the lemma follows from the second. As for the second, the inclusion

$$
f\left(x+\epsilon^{M} L\right) \subset f(x)+D_{x}\left(\epsilon^{M} L\right)
$$

follows from (10.3.2) and the fact that $\epsilon^{2 M} L \subset D_{x}\left(\epsilon^{M} L\right)$, a consequence of (10.3.1) and our hypothesis that $M>d$.

It remains only to prove the reverse inclusion, so let $y \in f(x)+D_{x}\left(\epsilon^{M} L\right)$. We need to find an element $x^{\prime} \in x+\epsilon^{M} L$ such that $f\left(x^{\prime}\right)=y$. We will obtain $x^{\prime}$ as the limit of a sequence $x=x_{0}, x_{1}, x_{2}, \ldots$ in $L$ constructed inductively so as to satisfy the two conditions

$$
\begin{aligned}
& x_{i}-x_{i-1} \in \epsilon^{M+i-1} L, \\
& y-f\left(x_{i}\right) \in \epsilon^{M+d+i} L
\end{aligned}
$$

for all $i \geq 1$. Some care is needed, because the first step is slightly different from all the remaining ones.

We begin by constructing $x_{1}$. Write $y$ as $f(x)+D_{x} h$ with $h \in \epsilon^{M} L$, and then put $x_{1}:=x+h$. Clearly (10.3.3) holds, and (10.3.4) follows from (10.3.2) and the hypothesis that $M>d$.

Now suppose that $i>1$ and that we have already constructed $x_{1}, x_{2}, \ldots, x_{i-1}$ satisfying (10.3.3) and (10.3.4). From (10.3.3) it follows that

$$
x_{i-1}-x \in \epsilon^{M} L \text {. }
$$

In particular $g\left(x_{i-1}\right) \equiv g(x) \bmod \epsilon^{M} \mathcal{O}$, and since $M>d$, we conclude that $d\left(x_{i-1}\right)=d$. Now applying (10.3.1) to $x_{i-1}$ rather than $x$, we see that

$$
\epsilon^{M+d+i-1} L \subset D_{x_{i-1}}\left(\epsilon^{M+i-1} L\right) .
$$

Using this together with (10.3.4) for $i-1$, we see that there exists $h_{i} \in \epsilon^{M+i-1} L$ such that

$$
D_{x_{i-1}} h_{i}=y-f\left(x_{i-1}\right) .
$$

Put $x_{i}:=x_{i-1}+h_{i}$. It is clear that (10.3.3) holds. It follows from (10.3.2) (with $M$ replaced by $M+i-1$ and $x$ replaced by $\left.x_{i-1}\right)$ and (10.3.6) that

$$
f\left(x_{i}\right) \equiv y \quad \bmod \epsilon^{2(M+i-1)} L .
$$

This yields (10.3.4) since $2(M+i-1) \geq M+d+i$ (use that $M>d$ and $i>1$ ). 
It is clear from (10.3.3) that the sequence $x_{i}$ has a limit $x^{\prime}$. It follows from (10.3.5) that $x^{\prime} \in x+\epsilon^{M} L$. Finally, we see from (10.3.4) that $f\left(x^{\prime}\right)=y$.

10.4. Images under $f$ of admissible subsets of $L$. Admissibility was discussed earlier in the context of a smooth scheme $X$ over $\mathcal{O}$. We are now interested in the case $X=\mathbb{A}^{n}$. Thus $X(\mathcal{O})=L$, and we have the notion of admissible subset in $L$. Moreover we continue with $f: L \rightarrow L$ as in the previous subsection.

Proposition 10.4.1. Let $M$ and e be non-negative integers, and let $Z$ be an $M$-admissible subset of $L$ such that $d(z) \leq e$ for all $z \in Z$. Then the subset $f(Z)$ is $N$-admissible, where $N$ is any positive integer large enough that $N>2 e$ and $N \geq M+e$.

Proof. This follows immediately from Lemma 10.3.1, applied not to the integer $M$, but to the integer $N-e$.

10.5. Fibers of $f_{N}: L / \epsilon^{N} L \rightarrow L / \epsilon^{N} L$. Let $N$ be a positive integer. Our given morphism $\mathbf{f}: \mathbb{A}^{n} \rightarrow \mathbb{A}^{n}$ induces a map

$$
f_{N}: L / \epsilon^{N} L \rightarrow L / \epsilon^{N} L
$$

on $\mathcal{O} / \epsilon^{N} \mathcal{O}$-valued points. There is a commutative square

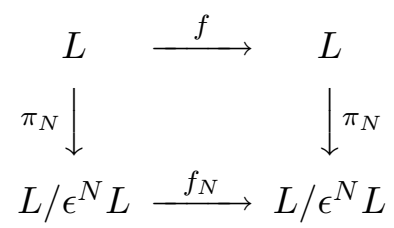

in which $\pi_{N}$ is the canonical surjection $L \rightarrow L / \epsilon^{N} L$.

We are interested in the fibers of $f_{N}$. In the linear case of subsection 10.2, as long as $N \geq d$, all fibers of $A_{N}$ are translates of $\operatorname{ker}\left(A_{N}\right)$, a vector space of dimension $d$ that is essentially independent of $N$ (see Lemma 10.2.2). Something similar happens in the non-linear case, with the role of $d$ being played by $d(x)$, but since $d(x)$ is no longer constant, the situation is necessarily more complicated. In order to analyze the fiber $f_{N}^{-1}(\bar{y})$ over a point $\bar{y} \in L / \epsilon^{N} L$ we will need to make an assumption (in part (2) of the next lemma) ensuring that $d(x)<N / 2$ for all points $x$ in $\pi_{N}^{-1}\left(f_{N}^{-1}(\bar{y})\right)$.

We will make use of the following definitions. For any non-negative integer $e$ we denote by $L_{\leq e}$ the subset of $L$ consisting of all points $x$ for which $d(x) \leq e$. 
The subset $L_{\leq e}$ of $L$ is obviously $(e+1)$-admissible. For $x \in L_{\leq e}$ and any integer $N$ such that $N \geq e$ we may apply Lemma 10.2.2 to the differential $D_{x}$ to conclude that $\operatorname{ker}\left(D_{x, N}\right)$ has dimension $d(x)$, is contained in $\epsilon^{N-e} L / \epsilon^{N} L \cong L / \epsilon^{e} L$, and is independent of $N$ when viewed as a subspace of $L / \epsilon^{e} L$. For $x \in L_{\leq e}$ we define

$$
V_{x}:=\operatorname{ker}\left(D_{x, e}\right)
$$

and we then have canonical isomorphisms

$$
V_{x} \cong \operatorname{ker}\left(D_{x, N}\right)
$$

for $N \geq e$.

Now suppose that $M>e$. Since $L_{\leq e}$ is $M$-admissible, it is the preimage of its image $\left(L / \epsilon^{M} L\right)_{\leq e}$ in $L / \epsilon^{M} L$. Since $D_{x, e}$ depends only on $\pi_{e}(x) \in L / \epsilon^{e} L$, so too does the linear subspace $V_{x}$ of $L / \epsilon^{e} L$, so that for any $z \in\left(L / \epsilon^{M} L\right)_{\leq e}$ we get a well-defined linear subspace $V_{z}$ of $L / \epsilon^{e} L$ by putting $V_{z}:=V_{x}$ for any $x \in L$ such that $\pi_{M}(x)=z$.

Lemma 10.5.1. Let $e, N$ be non-negative integers satisfying $N>2 e$. Put $M:=$ $N-e$, noting that $N \geq M>e$. Let $y \in L$ and put $\bar{y}:=\pi_{N}(y) \in L / \epsilon^{N} L$.

(1) For any $x \in f^{-1}(y) \cap L_{\leq e}$ the $d(x)$-dimensional affine linear subspace

$$
A_{x}:=\pi_{N}(x)+\epsilon^{M} V_{\pi_{M}(x)}
$$

of $L / \epsilon^{N} L$ is contained in $f_{N}^{-1}(\bar{y})$. Here $\epsilon^{M} V_{\pi_{M}(x)}$ is the $d(x)$-dimensional linear subspace of $\epsilon^{M} L / \epsilon^{N} L$ corresponding to $V_{\pi_{M}(x)}$ under the canonical isomorphism $\epsilon^{M} L / \epsilon^{N} L \cong L / \epsilon^{e} L$. Since the image of $\epsilon^{M} V_{\pi_{M}(x)}$ in $L / \epsilon^{M} L$ is 0 , the image of $A_{x}$ in $L / \epsilon^{M} L$ is the single point $\pi_{M}(x)$.

(2) If $f_{N}^{-1}(\bar{y})$ is contained in $\left(L / \epsilon^{N} L\right)_{\leq e}$, then $f^{-1}(y)$ is finite and

$$
f_{N}^{-1}(\bar{y})=\bigcup_{x \in f^{-1}(y)} A_{x} .
$$

(3) If the composed map

$$
f^{-1}(y) \hookrightarrow L \stackrel{\pi_{M}}{\longrightarrow} L / \epsilon^{M} L
$$

is injective, then $A_{x}, A_{x^{\prime}}$ are disjoint whenever $x, x^{\prime}$ are distinct points in $f^{-1}(y)$. 
Proof. (1) Let $x \in f^{-1}(y)$. Since $N>2 e$ implies $2 M \geq N$, equation (10.3.2) tells us that

$$
f(x+h) \equiv f(x)+D_{x} h \quad \bmod \epsilon^{N} L
$$

for all $h \in \epsilon^{M} L$. Using the canonical isomorphism $\epsilon^{M} L / \epsilon^{N} L \cong L / \epsilon^{e} L$, the restriction of $D_{x, N}$ to $\epsilon^{M} L / \epsilon^{N} L$ becomes identified with $D_{x, e}$, whose kernel is by definition $V_{x}=V_{\pi_{M}(x)}$. Therefore $A_{x}$ is contained in $f_{N}^{-1}(\bar{y})$.

(2) The morphism $\mathbf{f}$ is étale off the closed subscheme defined by the vanishing of the Jacobian $g$. It follows that for any $y^{\prime} \in L$ there are only finitely many points $x$ in the fiber $f^{-1}\left(y^{\prime}\right)$ for which $g(x) \neq 0$. Thus, the hypothesis made in (2) ensures the fiber $f^{-1}(y)$ is indeed finite.

Any element $\bar{x}^{\prime} \in f_{N}^{-1}(\bar{y})$ is represented by an element $x^{\prime} \in L$ such that

$$
f\left(x^{\prime}\right) \equiv y \quad \bmod \epsilon^{N} L .
$$

Our assumption that $f_{N}^{-1}(\bar{y})$ is contained in $\left(L / \epsilon^{N} L\right)_{\leq e}$ tells us that $d\left(x^{\prime}\right) \leq e$. Since $N>2 e$, Lemma 10.3.1 says that there exists $h \in \epsilon^{M} L$ such that $f\left(x^{\prime}-h\right)=$ $y$.

Put $x:=x^{\prime}-h$. Then $x \in f^{-1}(y)$ and we claim that $\bar{x}^{\prime} \in A_{x}$. Indeed, from (10.5.2) we see that

$$
f\left(x^{\prime}\right)=f(x+h) \equiv f(x)+D_{x} h \quad \bmod \epsilon^{N} L .
$$

Since $f(x)=y$ and $f\left(x^{\prime}\right) \equiv y \bmod \epsilon^{N} L,(10.5 .4)$ implies that $D_{x} h \in \epsilon^{N} L$, showing that $h$ represents an element in $\operatorname{ker}\left(D_{x, N}\right)=\epsilon^{M} V_{\pi_{M}(x)}$. Therefore $\bar{x}^{\prime} \in$ $A_{x}$.

(3) We have already noted that all points in $A_{x}$ have the same image as $x$ in $L / \epsilon^{M} L$. The injectivity of (10.5.1) then assures the disjointness of $A_{x}, A_{x^{\prime}}$ when $x, x^{\prime}$ are distinct points in $f^{-1}(y)$.

10.6. The vector bundle $V^{d}$. We retain all the notation of the previous subsection. In particular (for $M>e$ ) at each point $z \in\left(L / \epsilon^{M} L\right)_{\leq e}$ we have the vector space $V_{z}$, whose dimension depends on $z$. Now fix a non-negative integer $d$ such that $d \leq e$ and consider the $(e+1)$-admissible subset $L_{d}$ of $L$ consisting of all elements $x$ such that $d(x)=d$. Clearly $L_{d}$ is contained in $L_{\leq e}$. Since $L_{d}$ is also $M$-admissible, it is the preimage of its image $\left(L / \epsilon^{M} L\right)_{d}$ in $L / \epsilon^{M} L$. For each point $z \in\left(L / \epsilon^{M} L\right)_{d}$ the vector space $V_{z}$ is $d$-dimensional. 
We claim that we can assemble the vector spaces $V_{z}$ into a rank $d$ vector bundle $V^{d}$ over $\left(L / \epsilon^{M} L\right)_{d}$. Indeed, we just need to recall the general principle that, given a homomorphism of vector bundles over a scheme $Y$, and given a locally closed subset $Z$ of $Y$ over which the homomorphism has constant rank, the pointwise kernels of the homomorphism assemble into a vector bundle over $Z$. Here we are applying this general principle to the differential of $f_{M}: L / \epsilon^{M} L \rightarrow L / \epsilon^{M} L$, viewed as a homomorphism from the tangent bundle of $L / \epsilon^{M} L$ to itself.

Note that the particular choice of $M$ is unimportant, which is why we have omitted it from the notation. The smallest possible choice is $e+1$, so we get a vector bundle $V^{d}$ over $\left(L / \epsilon^{e+1} L\right)_{d}$, and its pullback by $\left(L / \epsilon^{M} L\right)_{d} \rightarrow\left(L / \epsilon^{e+1} L\right)_{d}$ gives us the vector bundle $V^{d}$ for $M$.

\section{Proof of Theorem 8.2 .2}

Among other things, we must show that $\mathbb{A}(\mathcal{O})_{s}$ is $N$-admissible when $N>2 e$ (abbreviating $e(w, r)$ to $e$ ). This follows from Proposition 10.4.1, once we note that the valuation of the Jacobian equals $e$ on $\mathfrak{t}_{w}(\mathcal{O})_{r}$ (see Lemma 8.2.1), and also that $\mathfrak{t}_{w}(\mathcal{O})_{r}$ is $(N-e)$-admissible (use Proposition 6.0.1). Here we used the nonemptiness of our stratum to conclude that for any root $\alpha$ we have $r(-\alpha)=r(\alpha)$ and hence

$$
r(\alpha) \leq \delta_{r} / 2 \leq e
$$

The rest of the proof is organized as follows. First we will prove the theorem in the case when $w=1$. Then we will deduce the general case from this special case.

11.1. Proof of Theorem 8.2.2 when $w=1$. We begin with some remarks comparing the strata $\mathbb{A}_{G}(\mathcal{O})_{s}$ for our reductive group $G$ with those for its derived group $G_{\text {der }}$. (We write $\mathbb{A}_{G}$ rather than $\mathbb{A}$ to emphasize the role of $G$.) We may then assume that $G$ is semisimple whenever it is convenient to do so.

Put $\mathfrak{z}:=\{u \in \mathfrak{t}: \alpha(u)=0 \quad \forall \alpha \in R\}$ and write $\mathfrak{t}_{\text {der }}$ for the Lie algebra of the maximal torus $T_{\text {der }}:=T \cap G_{\text {der }}$ of $G_{\text {der }}$. Since the index of connection of $R$ divides $|W|$ (see [Bou02, Ch. VI, no. 2.4, Prop. 7]) and is therefore invertible in $k$, we have $\mathfrak{t}=\mathfrak{z} \oplus \mathfrak{t}_{\text {der }}$. In fact, the simple coroots (for any choice of such) yield a $k$-basis for $\mathfrak{t}_{\text {der }}$, and the simple roots yield a $k$-basis for the $k$-dual of $\mathfrak{t}_{\text {der }}$. Dividing by $W$ to form adjoint quotients, we find that $\mathbb{A}_{G}(\mathcal{O})=\mathfrak{z}(\mathcal{O}) \times \mathbb{A}_{G_{\text {der }}}(\mathcal{O})$. 
The index set $\mathcal{S}$ for the strata is the same for $G_{\text {der }}$ as it is for $G$, and for $s \in \mathcal{S}$ we have $\mathbb{A}_{G}(\mathcal{O})_{s}=\mathfrak{z}(\mathcal{O}) \times \mathbb{A}_{G_{\text {der }}}(\mathcal{O})_{s}$. Therefore, when studying the strata $\mathbb{A}_{G}(\mathcal{O})_{s}$, it is harmless to assume that $G$ is semisimple. (For example, the codimension of $\mathbb{A}_{G_{\text {der }}}(\mathcal{O})_{s}$ in $\mathbb{A}_{G_{\text {der }}}(\mathcal{O})$ is the same as that of $\mathbb{A}_{G}(\mathcal{O})_{s}$ in $\mathbb{A}_{G}(\mathcal{O})$.)

In rest of this subsection we will always be taking $w=1$. Since we are only interested in non-empty strata, we only consider functions $r$ on $R$ taking values in the non-negative integers, and satisfying the property that

$$
R_{m}:=\{\alpha \in R: r(\alpha) \geq m\}
$$

be $\mathbb{Q}$-closed for all $m \geq 0$. In this subsection we write simply $\mathbb{A}(\mathcal{O})_{r}$ for the stratum in $\mathbb{A}(\mathcal{O})$ obtained as the image of $\mathfrak{t}(\mathcal{O})_{r}$. Of course $\mathbb{A}(\mathcal{O})_{x r}=\mathbb{A}(\mathcal{O})_{r}$ for all $x \in W$.

The theorem will be a simple consequence of the next two lemmas, the first involving scaling by $\epsilon^{m}$, the second involving reduction to a Levi subgroup.

In the first of the two lemmas we will need the following additional notation. For a non-negative integer $m$ we write $r+m$ for the function on $R$ whose value on a root $\alpha$ is $r(\alpha)+m$. Also we denote by $d_{i}$ the degree of the $i$-th basic invariant $f_{i}$.

Lemma 11.1.1. Suppose that $G$ is semisimple. Suppose further that $\mathbb{A}(\mathcal{O})_{r}$ is locally closed, irreducible, non-singular of codimension a in $\mathbb{A}(\mathcal{O})$. Then $\mathbb{A}(\mathcal{O})_{r+m}$ is locally closed, irreducible, non-singular of codimension $a+m\left(d_{1}+\cdots+d_{n}\right)$ in $\mathbb{A}(\mathcal{O})$.

Proof. Since $G$ is semisimple, we have $\mathfrak{t}(\mathcal{O})_{r+m}=\epsilon^{m} \mathfrak{t}(\mathcal{O})_{r}$. Therefore $\mathbb{A}(\mathcal{O})_{r+m}=$ $h \mathbb{A}(\mathcal{O})_{r}$, where $h$ is the $\mathcal{O}$-linear map from $L$ to $L$ defined by $\left(x_{1}, \ldots, x_{n}\right) \mapsto$ $\left(\epsilon^{m d_{1}} x_{1}, \ldots, \epsilon^{m d_{n}} x_{n}\right)$. Now use Lemma 10.2.1.

The second lemma involves the Levi subgroup $M$ of $G$ containing $T$ whose root system $R_{M}$ is equal to the $\mathbb{Q}$-closed subset $R_{1}=\{\alpha \in R: r(\alpha) \geq 1\}$ of the root system $R$. We need to consider $\mathbb{A}(\mathcal{O})$ for both $G$ and $M$, so to avoid confusion we now write $\mathbb{A}_{G}(\mathcal{O})$ and $\mathbb{A}_{M}(\mathcal{O})$. We write $r_{M}$ for the function on $R_{M}$ obtained by restriction from $r$.

Lemma 11.1.2. Suppose that $\mathbb{A}_{M}(\mathcal{O})_{r_{M}}$ is locally closed, irreducible, non-singular of codimension a in $\mathbb{A}_{M}(\mathcal{O})$. Then $\mathbb{A}_{G}(\mathcal{O})_{r}$ is locally closed, irreducible, nonsingular of codimension a in $\mathbb{A}_{G}(\mathcal{O})$. 
Proof. We write $W_{M}$ for the Weyl group of $M$. We will also need the subgroup $W_{M}^{\prime}$ of $W$ defined by

$$
W_{M}^{\prime}:=\left\{w \in W: w\left(R_{M}\right)=R_{M}\right\} .
$$

Note that $W_{M}$ is a normal subgroup of $W_{M}^{\prime}$. Since $\mathbb{A}_{M}$ is obtained by dividing $\mathfrak{t}$ by the action of the subgroup $W_{M}$ of $W$, there is an obvious surjective morphism

$$
g: \mathbb{A}_{M} \rightarrow \mathbb{A}_{G},
$$

and this yields a map

$$
g: \mathbb{A}_{M}(\mathcal{O}) \rightarrow \mathbb{A}_{G}(\mathcal{O}) .
$$

Note that $W_{M}^{\prime}$ acts on $\mathbb{A}_{M}$, and that the induced action on $\mathbb{A}_{M}(\mathcal{O})$ preserves the fibers of $g$.

Now define a polynomial function $Q$ on $\mathfrak{t}$ by

$$
Q=\prod_{\alpha \in R \backslash R_{M}} \alpha
$$

Since $Q$ is $W_{M}$-invariant (even $W_{M}^{\prime}$-invariant), we may also regard it as an element of the ring of regular functions on the affine variety $\mathbb{A}_{M}$. We denote by $\mathbb{A}_{M}^{b}$ the open $k$-subscheme of $\mathbb{A}_{M}$ obtained by removing the locus where $Q$ vanishes. Clearly $\mathbb{A}_{M}^{b}$ is stable under $W_{M}^{\prime}$. It follows from the discussion in subsection 2.3 that $Q$ is the square of the Jacobian of $g$ (up to some non-zero scalar in our base field $k$ ), and hence that the restriction $g^{b}$ of $g$ to $\mathbb{A}_{M}^{b}$ is étale.

Note that $\mathbb{A}_{M}^{b}(\mathcal{O})$ is the open, admissible (in fact 1-admissible) subset of $\mathbb{A}_{M}(\mathcal{O})$ consisting of all points $u \in \mathbb{A}_{M}(\mathcal{O})$ such that $Q(u)$ is a unit in $\mathcal{O}$. It follows easily from the definitions that

$$
\left(g^{b}\right)^{-1}\left(\mathbb{A}_{G}(\mathcal{O})_{r}\right)=\coprod_{x \in W_{M} \backslash W_{M}^{\prime}}\left(\mathbb{A}_{M}(\mathcal{O})_{x r_{M}} \cap \mathbb{A}_{M}^{b}(\mathcal{O})\right)
$$

and

$$
g^{b}\left(\mathbb{A}_{M}(\mathcal{O})_{r_{M}} \cap \mathbb{A}_{M}^{b}(\mathcal{O})\right)=\mathbb{A}_{G}(\mathcal{O})_{r} .
$$

We claim that for each $x \in W_{M}^{\prime}$ the subset $\mathbb{A}_{M}(\mathcal{O})_{x r_{M}} \cap \mathbb{A}_{M}^{b}(\mathcal{O})$ is locally closed, irreducible, non-singular of codimension $a$ in $\mathbb{A}_{M}^{b}(\mathcal{O})$. Indeed, using the action of $W_{M}^{\prime}$ on $\mathbb{A}_{M}(\mathcal{O})$, we may assume $x=1$, and then $\mathbb{A}_{M}(\mathcal{O})_{r_{M}} \cap \mathbb{A}_{M}^{b}(\mathcal{O})$, being open in $\mathbb{A}_{M}(\mathcal{O})_{r_{M}}$, inherits all the stated properties from $\mathbb{A}_{M}(\mathcal{O})_{r_{M}}$. (For 
irreducibility we need to remark that $\mathbb{A}_{M}(\mathcal{O})_{r_{M}} \cap \mathbb{A}_{M}^{b}(\mathcal{O})$ is non-empty, because by (11.1.2) it maps onto the non-empty set $\mathbb{A}_{G}(\mathcal{O})_{r}$.)

Next we claim that $\left(g^{b}\right)^{-1}\left(\mathbb{A}_{G}(\mathcal{O})_{r}\right)$ is locally closed and non-singular of codimension $a$ in $\mathbb{A}_{M}^{b}(\mathcal{O})$. Using (11.1.1) and the fact that each set $\mathbb{A}_{M}(\mathcal{O})_{x r_{M}} \cap$ $\mathbb{A}_{M}^{b}(\mathcal{O})$ is locally closed and non-singular of codimension $a$, we see that it is enough to show that when $x_{1}, x_{2}$ are distinct in $W_{M} \backslash W_{M}^{\prime}$, the closure of $\mathbb{A}_{M}(\mathcal{O})_{x_{1} r_{M}}$ in $\mathbb{A}_{M}(\mathcal{O})$ does not meet $\mathbb{A}_{M}(\mathcal{O})_{x_{2} r_{M}}$, and this follows from Lemma 11.1.3, applied to $M$ rather than $G$.

Now choose a positive integer $N$ so large that $\mathbb{A}_{G}(\mathcal{O})_{r}$ is $N$-admissible, and consider the commutative square

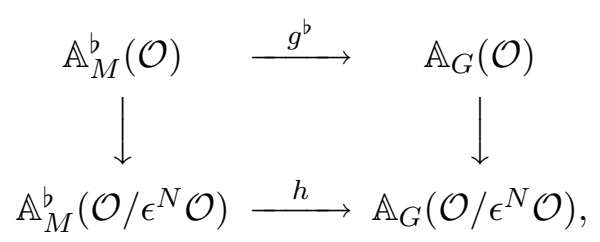

in which $h$ is obtained from $g^{b}$ by applying Greenberg's functor and is therefore étale (see 16.1). Since $\mathbb{A}_{G}(\mathcal{O})_{r}$ is $N$-admissible, it is the preimage of its image, call it $\mathbb{A}_{G}\left(\mathcal{O} / \epsilon^{N} \mathcal{O}\right)_{r}$, in $\mathbb{A}_{G}\left(\mathcal{O} / \epsilon^{N} \mathcal{O}\right)$. Since $h^{-1}\left(\mathbb{A}_{G}\left(\mathcal{O} / \epsilon^{N} \mathcal{O}\right)_{r}\right)$ has preimage $\left(g^{b}\right)^{-1}\left(\mathbb{A}_{G}(\mathcal{O})_{r}\right)$ in $\mathbb{A}_{M}^{b}(\mathcal{O})$, we conclude that $h^{-1}\left(\mathbb{A}_{G}\left(\mathcal{O} / \epsilon^{N} \mathcal{O}\right)_{r}\right)$ is locally closed and non-singular of codimension $a$ in $\mathbb{A}_{M}^{b}\left(\mathcal{O} / \epsilon^{N} \mathcal{O}\right)$. Since $h$ is étale, it is an open map, and moreover we know from (11.1.2) that $\mathbb{A}_{G}\left(\mathcal{O} / \epsilon^{N} \mathcal{O}\right)_{r}$ lies in the image of $h$. It then follows from Lemmas 13.1.2(1) and 13.2.1 that $\mathbb{A}_{G}\left(\mathcal{O} / \epsilon^{N} \mathcal{O}\right)_{r}$ is locally closed and non-singular. Since, again by (11.1.2), $\mathbb{A}_{G}\left(\mathcal{O} / \epsilon^{N} \mathcal{O}\right)_{r}$ is the image of an irreducible subset of $\mathbb{A}_{M}^{b}\left(\mathcal{O} / \epsilon^{N} \mathcal{O}\right)$, we conclude that $\mathbb{A}_{G}\left(\mathcal{O} / \epsilon^{N} \mathcal{O}\right)_{r}$ is irreducible. Since $h$ is étale, the $k$-schemes $\mathbb{A}_{G}\left(\mathcal{O} / \epsilon^{N} \mathcal{O}\right)_{r}$ and $h^{-1}\left(\mathbb{A}_{G}\left(\mathcal{O} / \epsilon^{N} \mathcal{O}\right)_{r}\right)$ have the same dimension. Moreover $\mathbb{A}_{G}\left(\mathcal{O} / \epsilon^{N} \mathcal{O}\right)$ and $\mathbb{A}_{M}^{b}\left(\mathcal{O} / \epsilon^{N} \mathcal{O}\right)$ have the same dimension, so the codimension of $\mathbb{A}_{G}\left(\mathcal{O} / \epsilon^{N} \mathcal{O}\right)_{r}$ in $\mathbb{A}_{G}\left(\mathcal{O} / \epsilon^{N} \mathcal{O}\right)$ is the same as that of $h^{-1}\left(\mathbb{A}_{G}\left(\mathcal{O} / \epsilon^{N} \mathcal{O}\right)_{r}\right)$ in $\mathbb{A}_{M}^{b}\left(\mathcal{O} / \epsilon^{N} \mathcal{O}\right)$, namely $a$. The lemma is proved.

Here is the lemma we needed in the previous proof. It involves two functions $r, r^{\prime}$ on $R$ taking values in the non-negative integers. As usual we assume that all the sets

$$
\begin{aligned}
& R_{m}=\{\alpha \in R: r(\alpha) \geq m\} \\
& R_{m}^{\prime}=\left\{\alpha \in R: r^{\prime}(\alpha) \geq m\right\}
\end{aligned}
$$


are $\mathbb{Q}$-closed.

Lemma 11.1.3. Assume that $\left|R_{m}\right|=\left|R_{m}^{\prime}\right|$ for all $m \geq 0$. If $\mathbb{A}_{G}(\mathcal{O})_{r}$ meets the closure of $\mathbb{A}_{G}(\mathcal{O})_{r^{\prime}}$ in $\mathbb{A}_{G}(\mathcal{O})$, then $r^{\prime} \in W r$.

Proof. Recall from before the integer

$$
\delta_{r}=\sum_{\alpha \in R} r(\alpha) .
$$

Our assumption that $\left|R_{m}\right|=\left|R_{m}^{\prime}\right|$ for all $m \geq 0$ means that $r, r^{\prime}$ have the same set (with multiplicities) of $|R|$ values. In particular $\delta_{r}=\delta_{r^{\prime}}$.

In this proof we will be using the scalar product

$$
\left(r_{1}, r_{2}\right):=\sum_{\alpha \in R} r_{1}(\alpha) r_{2}(\alpha)
$$

which is none other than the usual Euclidean inner product on $\mathbb{R}^{R}$. Since, when viewed as vectors in $\mathbb{R}^{R}, r$ and $r^{\prime}$ are permutations of each other, we have

$$
(r, r)=\left(r^{\prime}, r^{\prime}\right) .
$$

Pick $N \geq 0$ such that $r(\alpha) \leq N$ for all $\alpha \in R$. Define polynomials $Q_{r}, P_{r}$ on $\mathfrak{t}$ by

$$
\begin{aligned}
Q_{r} & :=\prod_{\alpha \in R} \alpha^{N-r(\alpha)} \\
P_{r} & :=\sum_{x \in W / W_{r}} Q_{x r} .
\end{aligned}
$$

Since $P_{r}$ has been defined so as to be $W$-invariant, it can also be thought of as a regular function on $\mathbb{A}_{G}$.

Suppose that $u \in \mathfrak{t}(\mathcal{O})_{r}$. Then

$$
\operatorname{val} Q_{x r}(u)=N \delta_{r}-(x r, r) .
$$

Since $(x r, x r)=(r, r)$, the Cauchy-Schwarz inequality implies that $(x r, r)<(r, r)$ when $x r \neq r$. Therefore

$$
\text { val } P_{r}(u)=N \delta_{r}-(r, r) .
$$

Now suppose that $u^{\prime} \in \mathfrak{t}(\mathcal{O})_{r^{\prime}}$. Then

$$
\operatorname{val} Q_{x r}\left(u^{\prime}\right)=N \delta_{r^{\prime}}-\left(x r, r^{\prime}\right) .
$$


Recall that $\delta_{r}=\delta_{r^{\prime}}$ and $(r, r)=\left(r^{\prime}, r^{\prime}\right)$. The Cauchy-Schwarz inequality implies that

$$
\operatorname{val} Q_{x r}\left(u^{\prime}\right) \geq N \delta_{r}-(r, r),
$$

with equality only if $x r=r^{\prime}$; therefore

$$
\text { val } P_{r}\left(u^{\prime}\right) \geq N \delta_{r}-(r, r),
$$

with equality only if $r^{\prime} \in W r$. Thus, if $r^{\prime} \notin W r$, the admissible open subset

$$
\left\{v \in \mathbb{A}_{G}(\mathcal{O}): \operatorname{val} P_{r}(v) \leq N \delta_{r}-(r, r)\right\}
$$

of $\mathbb{A}_{G}(\mathcal{O})$ contains $\mathbb{A}_{G}(\mathcal{O})_{r}$ and is disjoint from $\mathbb{A}_{G}(\mathcal{O})_{r^{\prime}}$.

Now we can prove Theorem 8.2.2 when $w=1$. One of the assertions of the theorem is that $\mathbb{A}_{G}(\mathcal{O})_{r}$ has codimension

$$
D_{G}(r):=d_{G}(r)+\frac{1}{2}\left(\delta_{r}+c_{w}\right)
$$

in $\mathbb{A}_{G}(\mathcal{O})$, where $d_{G}(r)$ denotes the codimension of $\mathfrak{t}(\mathcal{O})_{r}$ in $\mathfrak{t}(\mathcal{O})$. Since $c_{w}=0$ when $w=1$, we can write $D_{G}(r)$ more simply as

$$
D_{G}(r)=d_{G}(r)+\sum_{\alpha \in R^{+}} r(\alpha) .
$$

We begin by proving parts (1) and (2) of the theorem. We do this by induction on $|R|$, the case when $|R|=0$ being trivial. Now we do the induction step. First suppose that 0 actually occurs as a value of $r$, so that $R_{1}$ is strictly smaller than $R$. Thus the theorem holds for the group $M$ in Lemma 11.1.2 by our inductive hypothesis. Therefore Lemma 11.1.2 implies that $\mathbb{A}_{G}(\mathcal{O})_{r}$ is locally closed, irreducible, non-singular of codimension $D_{M}\left(r_{M}\right)$ in $\mathbb{A}_{G}(\mathcal{O})$. It remains to check that $D_{M}\left(r_{M}\right)=D_{G}(r)$, but this is clear, since $r$ vanishes on roots of $G$ that are not roots of $M$. (Note that $\mathfrak{t}(\mathcal{O})_{r}$ is open in $\mathfrak{t}(\mathcal{O})_{r_{M}}$, so that $d_{G}(r)=d_{M}\left(r_{M}\right)$.)

Now suppose that 0 does not occur as a value of $r$, and let $m$ be the smallest integer which actually does occur as a value of $r$. Then $r$ can be written as $r^{\prime}+m$, and 0 occurs as a value of $r^{\prime}$, so that the theorem holds for $r^{\prime}$ by what we have already proved. After replacing $G$ by its derived group, we may assume that $G$ is semisimple and apply Lemma 11.1.1 to conclude that $\mathbb{A}_{G}(\mathcal{O})_{r}$ is locally closed, irreducible, non-singular of codimension $D_{G}\left(r^{\prime}\right)+m\left(d_{1}+\cdots+d_{n}\right)$ in $\mathbb{A}_{G}(\mathcal{O})$. It follows easily from (2.3.2) that $D_{G}\left(r^{\prime}\right)+m\left(d_{1}+\cdots+d_{n}\right)=D_{G}(r)$, and the proof 
of parts (1) and (2) of the theorem is now complete. (Note that we obtain a set of basic invariants for $G$ by taking those for $G_{\text {der }}$ together with any $k$-basis for the $k$-dual of $\mathfrak{z}$, these additional ones obviously having degree 1 . However when we wrote $d_{1}+\cdots+d_{n}$ in the preceding lines, we meant the sum of the degrees of the basic invariants for $G_{\text {der }}$.)

It remains to prove part (3) of the theorem, which asserts that $\mathfrak{t}(\mathcal{O})_{r}$ is smooth over $\mathbb{A}(\mathcal{O})_{r}$. Since $\mathfrak{t}(\mathcal{O})_{r}$ and $\mathbb{A}(\mathcal{O})_{r}$ are non-singular, it suffices (see Lemma 5.4.1) to check that for each $u \in \mathfrak{t}(\mathcal{O})_{r}$ the differential $d f_{u}$ of $f$ maps $T_{\mathfrak{t}(\mathcal{O})_{r}, u}$ onto $T_{\mathbb{A}(\mathcal{O})_{r}, f(u)}$. For this we just need to show that $T_{\mathbb{A}(\mathcal{O})_{r}, f(u)}$ and the image under $d f_{u}$ of $T_{\mathfrak{t}(\mathcal{O})_{r}, u}$ have the same codimension in $T_{\mathbb{A}(\mathcal{O}), f(u)}$. From part (2) of the theorem we know that the codimension of $T_{\mathbb{A}(\mathcal{O})_{r}, f(u)}$ in $T_{\mathbb{A}(\mathcal{O}), f(u)}$ is

$$
d_{G}(r)+\sum_{\alpha \in R^{+}} r(\alpha)
$$

Since the valuation of the determinant of $d f_{u}$ is $\sum_{\alpha \in R^{+}} r(\alpha)$ (see (2.3.1)), and the codimension of the tangent space $T_{\mathfrak{t}(\mathcal{O})_{r}, u}$ in $T_{\mathfrak{t}(\mathcal{O}), u}$ is equal to $d_{G}(r)$, we conclude that the codimension of the image under $d f_{u}$ of $T_{\mathfrak{t}(\mathcal{O})_{r}, u}$ is also equal to the expression (11.1.3), and we are done.

11.2. Proof of Theorem 8.2.2 in general. Now we prove the theorem in the general case. So consider a pair $(w, r)$ such that $\mathbb{A}(\mathcal{O})_{w, r}$ is non-empty, and let $s$ denote the $W$-orbit of $(w, r)$.

Here is the idea of the proof. As usual we denote by $E$ the field $F_{l}$, with $l=o(w)$. We once again denote by $r_{E}$ the integer valued function on $R$ obtained by multiplying $r$ by $l$. From the special case of the theorem that we have already proved (applied to $E$ rather than $F$ ), we understand $\mathfrak{t}\left(\mathcal{O}_{E}\right)_{r_{E}}$ and $\mathbb{A}\left(\mathcal{O}_{E}\right)_{r_{E}}$, and we are going to deduce the theorem in general by taking fixed points of suitable automorphisms of order $l$.

In the case of $\mathfrak{t}\left(\mathcal{O}_{E}\right)_{r_{E}}$, we consider the automorphism $u \mapsto w \tau_{E}(u)$. The fixed point set of this action is of course $\mathfrak{t}_{w}(\mathcal{O})_{r}$. In the case of $\mathbb{A}\left(\mathcal{O}_{E}\right)_{r_{E}}$, we consider the action of $\tau_{E}$. The fixed point set of this action contains $\mathbb{A}(\mathcal{O})_{w, r}$. These two automorphisms give us actions of the cyclic group $\mathbb{Z} / l \mathbb{Z}$, and the map

$$
f: \mathfrak{t}\left(\mathcal{O}_{E}\right)_{r_{E}} \rightarrow \mathbb{A}\left(\mathcal{O}_{E}\right)_{r_{E}}
$$


is $\mathbb{Z} / l \mathbb{Z}$-equivariant. Taking fixed points under $\mathbb{Z} / l \mathbb{Z}$, we get

$$
\mathfrak{t}_{w}(\mathcal{O})_{r} \stackrel{f_{w}}{\longrightarrow} \mathbb{A}(\mathcal{O})_{s} \hookrightarrow \mathbb{A}\left(\mathcal{O}_{E}\right)_{r_{E}}^{\mathbb{Z} / l \mathbb{Z}}
$$

Since $l$ is invertible in $k$, taking fixed points under $\mathbb{Z} / l \mathbb{Z}$ preserves non-singularity and smoothness, as is discussed in appendix 15. This will end up giving us a good handle on $\mathbb{A}(\mathcal{O})_{s}$. Unfortunately we cannot apply appendix 15 directly to (11.2.1), since we need to be dealing with schemes of finite type over $k$. To achieve this we use that all the sets under consideration are $N$-admissible for sufficiently large $N$.

More precisely there are four admissible sets under consideration. We begin by choosing $M$ large enough that $r(\alpha)<M$ for all $\alpha \in R$. This guarantees (see Proposition 6.0.1) that $\mathfrak{t}_{w}(\mathcal{O})_{r}$ and $\mathfrak{t}\left(\mathcal{O}_{E}\right)_{r_{E}}$ are $M$-admissible. Increasing $M$ as need be, we may also assume that $\mathbb{A}(\mathcal{O})_{s}$ and $\mathbb{A}\left(\mathcal{O}_{E}\right)_{r_{E}}$ are $M$-admissible. Now let $N$ be any integer such that $N \geq M$.

Thus, now letting $\mathfrak{t}_{w}\left(\mathcal{O} / \epsilon^{N} \mathcal{O}\right)_{r}$ denote the image of $\mathfrak{t}_{w}(\mathcal{O})_{r}$ under $\mathfrak{t}_{w}(\mathcal{O}) \rightarrow$ $\mathfrak{t}_{w}\left(\mathcal{O} / \epsilon^{N} \mathcal{O}\right)$, the set $\mathfrak{t}_{w}(\mathcal{O})_{r}$ is the preimage of $\mathfrak{t}_{w}\left(\mathcal{O} / \epsilon^{N} \mathcal{O}\right)_{r}$. Similarly, letting $\mathfrak{t}\left(\mathcal{O}_{E} / \epsilon^{N} \mathcal{O}_{E}\right)_{r_{E}}$ denote the image of $\mathfrak{t}\left(\mathcal{O}_{E}\right)_{r_{E}}$ under $\mathfrak{t}\left(\mathcal{O}_{E}\right) \rightarrow \mathfrak{t}\left(\mathcal{O}_{E} / \epsilon^{N} \mathcal{O}_{E}\right)$, the set $\mathfrak{t}\left(\mathcal{O}_{E}\right)_{r_{E}}$ is the preimage of $\mathfrak{t}\left(\mathcal{O}_{E} / \epsilon^{N} \mathcal{O}_{E}\right)_{r_{E}}$.

In addition $\mathbb{A}(\mathcal{O})_{s}$ is the preimage of its image $\mathbb{A}\left(\mathcal{O} / \epsilon^{N} \mathcal{O}\right)_{s}$ in $\mathbb{A}\left(\mathcal{O} / \epsilon^{N} \mathcal{O}\right)$, and similarly $\mathbb{A}\left(\mathcal{O}_{E}\right)_{r_{E}}$ is the preimage of its image $\mathbb{A}\left(\mathcal{O}_{E} / \epsilon^{N} \mathcal{O}_{E}\right)_{r_{E}}$ in $\mathbb{A}\left(\mathcal{O}_{E} / \epsilon^{N} \mathcal{O}_{E}\right)$. With all this notation in place, we can now finish the proof.

As noted in subsection 16.3, there are two different ways to use Greenberg's functor to regard $\mathfrak{t}\left(\mathcal{O}_{E} / \epsilon^{N} \mathcal{O}_{E}\right)$ as the set of $k$-points of a $k$-scheme. One is to apply Greenberg's functor directly to $\mathfrak{t}$, but working with $\mathcal{O}_{E}$ rather than $\mathcal{O}$. The other is to apply restriction of scalars $R_{\mathcal{O}_{E} / \mathcal{O}}$ to $\mathfrak{t}$ and then use Greenberg's functor for $\mathcal{O}$. Fortunately, 16.3 assures us that the two methods give the same result, so we will be free to use whichever interpretation is most convenient at a given moment. The same remarks apply to $\mathbb{A}\left(\mathcal{O}_{E} / \epsilon^{N} \mathcal{O}_{E}\right)$.

Consider the commutative square

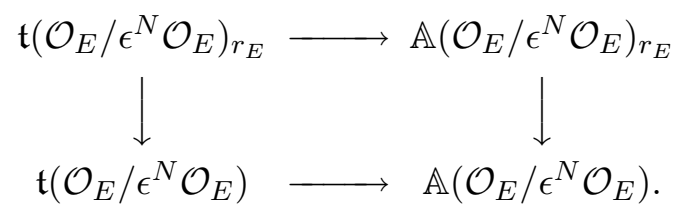


From Proposition 6.0.1 and the special case of the theorem that has already been proved, we know that the vertical arrows are locally closed immersions, that the top horizontal arrow is smooth, and that all four corners of the square are non-singular.

Recall from 4.4 the $\mathbb{Z} / l \mathbb{Z}$-action on $R_{\mathcal{O}_{E} / \mathcal{O}^{\mathfrak{t}}}$ whose fixed point scheme is $\mathfrak{t}_{w}$. From it we get an action of $\mathbb{Z} / l \mathbb{Z}$ on the $k$-scheme

$$
\left(R_{\mathcal{O}_{E} / \mathcal{O}} \mathfrak{t}\right)\left(\mathcal{O} / \epsilon^{N} \mathcal{O}\right)=\mathfrak{t}\left(\mathcal{O}_{E} / \epsilon^{N} \mathcal{O}_{E}\right)
$$

It follows from Proposition 4.8.2(3) that our action preserves $\mathfrak{t}\left(\mathcal{O}_{E} / \epsilon^{N} \mathcal{O}_{E}\right)_{r_{E}}$ settheoretically, hence scheme-theoretically as well, since we are using the induced reduced subscheme structure. Similarly, $\mathbb{Z} / l \mathbb{Z}$ acts on $\mathbb{A}\left(\mathcal{O}_{E} / \epsilon^{N} \mathcal{O}_{E}\right)$, preserving the locally closed subscheme $\mathbb{A}\left(\mathcal{O}_{E} / \epsilon^{N} \mathcal{O}_{E}\right)_{r_{E}}$.

Now we take fixed points under $\mathbb{Z} / l \mathbb{Z}$ everywhere in the commutative square we are considering. Bearing in mind that taking fixed points commutes with Greenberg's functor (see subsection 16.4), we obtain the commutative square

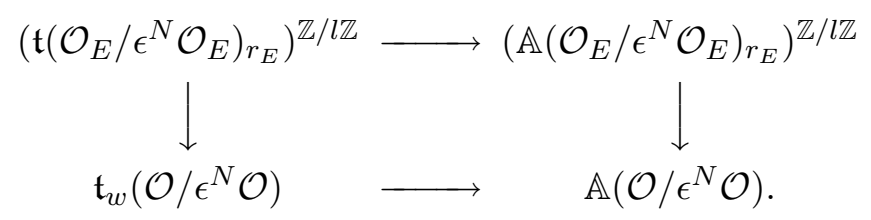

Since taking $\mathbb{Z} / l \mathbb{Z}$-fixed points preserves immersions, non-singularity, and smoothness (see Lemma 15.4.2), we conclude that all four corners of our square are non-singular, that the top horizontal arrow is smooth, and that the two vertical arrows are locally closed immersions.

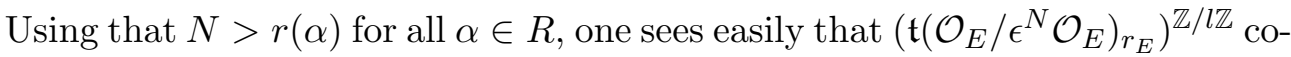
incides with $\mathfrak{t}_{w}\left(\mathcal{O} / \epsilon^{N} \mathcal{O}\right)_{r}$ set-theoretically. Since both are non-singular schemes, hence reduced, they actually coincide as subschemes.

The image of $\left(\mathfrak{t}\left(\mathcal{O}_{E} / \epsilon^{N} \mathcal{O}_{E}\right)_{r_{E}}\right)^{\mathbb{Z} / l \mathbb{Z}}=\mathfrak{t}_{w}\left(\mathcal{O} / \epsilon^{N} \mathcal{O}\right)_{r}$ in $\left(\mathbb{A}\left(\mathcal{O}_{E} / \epsilon^{N} \mathcal{O}_{E}\right)_{r_{E}}\right)^{\mathbb{Z} / l \mathbb{Z}}$ is open (since the top horizontal arrow is smooth) and its further image in $\mathbb{A}\left(\mathcal{O} / \epsilon^{N} \mathcal{O}\right)$, namely $\mathbb{A}\left(\mathcal{O} / \epsilon^{N} \mathcal{O}\right)_{s}$, is therefore locally closed in $\mathbb{A}\left(\mathcal{O} / \epsilon^{N} \mathcal{O}\right)$. At the same time we see that $\mathbb{A}\left(\mathcal{O} / \epsilon^{N} \mathcal{O}\right)_{s}$ is non-singular and that

$$
\mathfrak{t}_{w}\left(\mathcal{O} / \epsilon^{N} \mathcal{O}\right)_{r} \rightarrow \mathbb{A}\left(\mathcal{O} / \epsilon^{N} \mathcal{O}\right)_{s}
$$


is smooth. Since we have proved that (11.2.2) is smooth for all $N \geq M$, we conclude that

$$
\mathfrak{t}_{w}(\mathcal{O})_{r} \rightarrow \mathbb{A}(\mathcal{O})_{s}
$$

is smooth. Since $\mathfrak{t}_{w}\left(\mathcal{O} / \epsilon^{N} \mathcal{O}\right)_{r}$ is irreducible, so too is $\mathbb{A}\left(\mathcal{O} / \epsilon^{N} \mathcal{O}\right)_{s}$.

At this point we have proved all parts of the theorem except for the statement concerning the codimension of $\mathbb{A}(\mathcal{O})_{s}$. For this we use tangent spaces (which we are free to use since we now know that the admissible subsets $\mathfrak{t}_{w}(\mathcal{O})_{r}$ and $\mathbb{A}(\mathcal{O})_{s}$ are locally closed and non-singular). Choose some point $u \in \mathfrak{t}_{w}(\mathcal{O})_{r}$ and let $c$ denote its image in $\mathbb{A}(\mathcal{O})_{s}$. The codimension of $\mathbb{A}(\mathcal{O})_{s}$ in $\mathbb{A}(\mathcal{O})$ is the same as that of the tangent space to $\mathbb{A}(\mathcal{O})_{s}$ at $c$ in the tangent space to $\mathbb{A}(\mathcal{O})$ at $c$. Now, since (11.2.3) is smooth, Lemma 5.4.1 tells us that the tangent space to $\mathfrak{t}_{w}(\mathcal{O})_{r}$ at $u$ maps onto the tangent space to $\mathbb{A}(\mathcal{O})_{s}$ at $c$.

We conclude that the codimension of $\mathbb{A}(\mathcal{O})_{s}$ in $\mathbb{A}(\mathcal{O})$ is the sum of the codimension of $\mathfrak{t}_{w}(\mathcal{O})_{r}$ in $\mathfrak{t}_{w}(\mathcal{O})$ (a number we have denoted by $d(w, r)$ ) and the valuation of the Jacobian of $f_{w}$ at the point $u$ (which by Lemma 8.2.1 we know to be equal to $\left.e(w, r)=\left(\delta_{r}+c_{w}\right) / 2\right)$. This finally finishes the proof of the theorem.

\section{Proof of Theorem 9.1.1}

The idea of the proof is simple enough. We will check that Lemma 10.5.1 applies to our situation, concluding that each fiber of the morphism $\mathfrak{t}_{w}\left(\mathcal{O} / \epsilon^{N} \mathcal{O}\right)_{r} \rightarrow$ $\mathbb{A}\left(\mathcal{O} / \epsilon^{N} \mathcal{O}\right)_{s}$ is a disjoint union of affine spaces of dimension $e$. These affine spaces are permuted simply transitively by $W_{w, r}$, and we have already proved that the morphism is smooth. This makes it plausible that the theorem is true, but we must construct the rank $e$ vector bundle $\tilde{V}$ and check that the morphism really is a torsor for $H=W_{w, r} \times \tilde{V}$.

Let $c \in \mathbb{A}(\mathcal{O})_{s}$ and let $\bar{c}$ denote the image of $c$ in $\mathbb{A}\left(\mathcal{O} / \epsilon^{N} \mathcal{O}\right)_{s}$. Lemma 10.5.1 will give us information about the fiber $Z$ of the morphism

$$
f_{w, N}: \mathfrak{t}_{w}\left(\mathcal{O} / \epsilon^{N} \mathcal{O}\right) \rightarrow \mathbb{A}\left(\mathcal{O} / \epsilon^{N} \mathcal{O}\right)
$$

over the point $\bar{c}$.

We need to check that the hypotheses of the lemma are verified. As in that lemma we will use $L$ to denote $\mathcal{O}^{n}=\mathbb{A}(\mathcal{O})$. We are assuming that $N>2 e$, so we just need to verify the assumptions made in (2) and (3) of the lemma. 
By Lemma 7.3.2 we have

$$
f_{w}^{-1}\left(\mathbb{A}(\mathcal{O})_{s}\right)=\coprod_{x \in W_{w} / W_{w, r}} \mathfrak{t}_{w}(\mathcal{O})_{x r} .
$$

It then follows from Lemma 8.2.1 that val det $d f_{w}$ takes the constant value $e$ on $f_{w}^{-1}\left(\mathbb{A}(\mathcal{O})_{s}\right)$. Since $\mathbb{A}(\mathcal{O})_{s}$ is $N$-admissible, the preimage of $Z$ in $\mathfrak{t}_{w}(\mathcal{O})$ is contained in $f_{w}^{-1}\left(\mathbb{A}(\mathcal{O})_{s}\right)$, and therefore val $\operatorname{det} d f_{w}$ takes the constant value $e$ on that preimage, showing that the assumption about $f_{w, N}^{-1}(\bar{c})$ made in (2) of Lemma 10.5.1 does hold. Note that part (1) of that lemma is applicable as well.

As for the assumption on the fiber $f_{w}^{-1}(c)$ made in (3) of Lemma 10.5.1, we first recall (see 7.2) that the group $W_{w}$ acts simply transitively on this fiber. Next, recall from the first paragraph of section 11 that

$$
r(\alpha) \leq e<N-e \quad \forall \alpha \in R
$$

and hence that $\mathfrak{t}_{w}(\mathcal{O})_{r}$ is $(N-e)$-admissible. Since (see 4.7 and use (12.0.5)) $W_{w}$ acts freely on the image of this fiber in $\mathfrak{t}_{w}\left(\mathcal{O} / \epsilon^{N-e} \mathcal{O}\right)$, we conclude that the fiber injects into $\mathfrak{t}_{w}\left(\mathcal{O} / \epsilon^{N-e} \mathcal{O}\right)$, as desired.

The lemma then describes the fiber $Z=f_{w, N}^{-1}(\bar{c})$ as a disjoint union of affine spaces $A_{u}$, one for each $u \in f_{w}^{-1}(c)$. However, we are really interested in the fiber $g^{-1}(\bar{c})$ of the morphism

$$
g: \mathfrak{t}_{w}\left(\mathcal{O} / \epsilon^{N} \mathcal{O}\right)_{r} \rightarrow \mathbb{A}\left(\mathcal{O} / \epsilon^{N} \mathcal{O}\right)_{s}
$$

obtained by restriction from $f_{w, N}$. For each $u \in f_{w}^{-1}(c)$ there exists (by (12.0.4)) $x \in W_{w}$ such that $u \in \mathfrak{t}_{w}(\mathcal{O})_{x r}$. We noted in part (1) of Lemma 10.5.1 that all the points in $A_{u}$ have the same image as $u$ in $\mathfrak{t}_{w}\left(\mathcal{O} / \epsilon^{N-e} \mathcal{O}\right)$. Since $\mathfrak{t}_{w}(\mathcal{O})_{x r}$ is $(N-e)$-admissible, it follows that $A_{u} \subset \mathfrak{t}_{w}\left(\mathcal{O} / \epsilon^{N} \mathcal{O}\right)_{x r}$. Therefore

$$
g^{-1}(\bar{c})=Z \cap \mathfrak{t}_{w}\left(\mathcal{O} / \epsilon^{N} \mathcal{O}\right)_{r}=\coprod_{u} A_{u},
$$

where the index set for the disjoint union is $f_{w}^{-1}(c) \cap \mathfrak{t}_{w}(\mathcal{O})_{r}$. From Lemma 7.3.2 we know that $W_{w, r}$ acts simply transitively on $f_{w}^{-1}(c) \cap \mathfrak{t}_{w}(\mathcal{O})_{r}$. Thus the natural action of $W_{w, r}$ on $g^{-1}(\bar{c})$ permutes simply transitively the $e$-dimensional affine spaces $A_{u}$ appearing in the disjoint union (12.0.6).

These affine spaces arise as orbits of translation actions of certain vector spaces described in Lemma 10.5.1. We are going to use the discussion in 10.6 to assemble these vector spaces into a vector bundle. Eventually we will arrive at the vector 
bundle $\tilde{V}$, but we must begin with the one (over a different base space) that is provided by 10.6 .

Put $M:=N-e$. We have already noted that $\mathfrak{t}_{w}(\mathcal{O})_{r}$ is $M$-admissible. Therefore the obvious surjection

$$
\pi: \mathfrak{t}_{w}\left(\mathcal{O} / \epsilon^{N} \mathcal{O}\right)_{r} \rightarrow \mathfrak{t}_{w}\left(\mathcal{O} / \epsilon^{M} \mathcal{O}\right)_{r}
$$

is an affine space bundle, more precisely, a torsor (actually trivial, not that it matters) under the vector group

$$
\operatorname{ker}\left[\mathfrak{t}_{w}\left(\mathcal{O} / \epsilon^{N} \mathcal{O}\right) \rightarrow \mathfrak{t}_{w}\left(\mathcal{O} / \epsilon^{M} \mathcal{O}\right)\right]=\mathfrak{t}_{w}\left(\mathcal{O} / \epsilon^{e} \mathcal{O}\right)
$$

(the identification being made using multiplication by $\epsilon^{M}$ ).

Since val det $d f_{w}$ takes the constant value $e$ on $\mathfrak{t}_{w}(\mathcal{O})_{r}$, subsection 10.6 provides us with a rank $e$ vector bundle $V$ over $\mathfrak{t}_{w}\left(\mathcal{O} / \epsilon^{M} \mathcal{O}\right)_{r}$, obtained by restriction from the vector bundle $V^{e}$ of 10.6. In fact $V$ is a subbundle of the constant vector bundle over $\mathfrak{t}_{w}\left(\mathcal{O} / \epsilon^{M} \mathcal{O}\right)_{r}$ with fiber (12.0.7). It is clear from its definition that $V$ is $W_{w, r}$-equivariant with respect to the natural action of $W_{w, r}$ on $\mathfrak{t}_{w}\left(\mathcal{O} / \epsilon^{M} \mathcal{O}\right)_{r}$.

The vector bundle $V$ acts by translations on the affine space bundle $\mathfrak{t}_{w}\left(\mathcal{O} / \epsilon^{N} \mathcal{O}\right)_{r}$ over $\mathfrak{t}_{w}\left(\mathcal{O} / \epsilon^{M} \mathcal{O}\right)_{r}$, and we may divide out by its action, obtaining a factorization

$$
\mathfrak{t}_{w}\left(\mathcal{O} / \epsilon^{N} \mathcal{O}\right)_{r} \stackrel{\rho}{\rightarrow} \mathfrak{t}_{w}\left(\mathcal{O} / \epsilon^{N} \mathcal{O}\right)_{r} / V \stackrel{\eta}{\rightarrow} \mathfrak{t}_{w}\left(\mathcal{O} / \epsilon^{M} \mathcal{O}\right)_{r}
$$

of $\pi$, in which $\rho, \eta$ are both affine space bundles. More precisely $\rho$ is a torsor for $\eta^{*} V$, and $\eta$ is a torsor for the vector bundle obtained by taking the quotient of the constant vector bundle $\mathfrak{t}_{w}\left(\mathcal{O} / \epsilon^{e} \mathcal{O}\right)$ by its subbundle $V$.

By Lemma 10.5.1 the morphism $g$ is constant on the fibers of the bundle $\rho$. By faithfully flat descent we see that $g$ factors uniquely as

$$
\mathfrak{t}_{w}\left(\mathcal{O} / \epsilon^{N} \mathcal{O}\right)_{r} \stackrel{\rho}{\rightarrow} \mathfrak{t}_{w}\left(\mathcal{O} / \epsilon^{N} \mathcal{O}\right)_{r} / V \stackrel{h}{\rightarrow} \mathbb{A}\left(\mathcal{O} / \epsilon^{N} \mathcal{O}\right)_{s} .
$$

(To apply descent theory we just need to check the equality of two morphisms $B \rightarrow \mathbb{A}\left(\mathcal{O} / \epsilon^{N} \mathcal{O}\right)_{s}$, where $B$ denotes the fiber product of $\mathfrak{t}_{w}\left(\mathcal{O} / \epsilon^{N} \mathcal{O}\right)_{r}$ with itself over $\mathfrak{t}_{w}\left(\mathcal{O} / \epsilon^{N} \mathcal{O}\right)_{r} / V$. Now $B$, being itself an affine space bundle over the reduced scheme $\mathfrak{t}_{w}\left(\mathcal{O} / \epsilon^{M} \mathcal{O}\right)_{r}$, is also reduced, so that the equality of our two morphisms $B \rightarrow \mathbb{A}\left(\mathcal{O} / \epsilon^{N} \mathcal{O}\right)_{s}$ follows from the obvious fact that they coincide on $k$-points.)

Now $g$ is smooth (by Theorem 8.2.2) and so is $\rho$; therefore $h$ is smooth as well. The $W_{w, r}$-equivariance of $V$ ensures that the action of $W_{w, r}$ on $\mathfrak{t}_{w}\left(\mathcal{O} / \epsilon^{N} \mathcal{O}\right)_{r}$ descends to an action on $\mathfrak{t}_{w}\left(\mathcal{O} / \epsilon^{N} \mathcal{O}\right)_{r} / V$ over $\mathbb{A}\left(\mathcal{O} / \epsilon^{N} \mathcal{O}\right)_{s}$, and Lemma 10.5.1 
tells us that $W_{w, r}$ acts simply transitively on the fibers of $h$. This means that $h$ is in fact étale, and hence that $\mathfrak{t}_{w}\left(\mathcal{O} / \epsilon^{N} \mathcal{O}\right)_{r} / V$ is a $W_{w, r}$-torsor over $\mathbb{A}\left(\mathcal{O} / \epsilon^{N} \mathcal{O}\right)_{s}$.

The pullback $\eta^{*} V$ is a $W_{w, r}$-equivariant vector bundle over $\mathfrak{t}_{w}\left(\mathcal{O} / \epsilon^{N} \mathcal{O}\right)_{r} / V$. Since $h$ is a $W_{w, r}$-torsor, $\eta^{*} V$ descends to a vector bundle $\tilde{V}$ on $\mathbb{A}\left(\mathcal{O} / \epsilon^{N} \mathcal{O}\right)_{s}$, and we see from the factorization $g=h \rho$ that $g$ is a $\left(W_{w, r} \times \tilde{V}\right)$-torsor. The proof is now complete.

\section{Appendix. Technichl lemmas Related to ADmissibility}

In this appendix we verify some lemmas needed to back up the statements we made in section 5 concerning admissible subsets of $X(\mathcal{O})$.

\subsection{Elementary facts about open mappings.}

Lemma 13.1.1. Let $f: Y \rightarrow X$ be a continuous map of topological spaces. Then the following three conditions are equivalent:

(1) $f$ is an open mapping.

(2) For every closed subset $Z \subset Y$ the set $\left\{x \in X: f^{-1}(x) \subset Z\right\}$ is closed in $X$.

(3) For every subset $S \subset X$ we have $\overline{f^{-1}(S)}=f^{-1}(\bar{S})$. Here the overlines indicate closures.

Proof. (1) holds iff $f(U)$ is open for every open $U \subset Y$. Phrasing this in complementary terms, (1) holds iff $f\left(Z^{c}\right)^{c}$ is closed for every closed subset $Z \subset Y$, where the superscript $c$ indicates complement. Since $f\left(Z^{c}\right)^{c}=\left\{x \in X: f^{-1}(x) \subset Z\right\}$, we see that (1) is equivalent to (2).

Now consider (3). Since $f$ is continuous, $f^{-1} \bar{S}$ is a closed subset containing $f^{-1} S$. (In order to lighten the notation we often write, for example, $f^{-1} S$ rather than $f^{-1}(S)$.) Therefore (3) holds iff for every $S \subset X$ and every closed $Z \subset Y$ we have the implication $Z \supset f^{-1} S \Longrightarrow Z \supset f^{-1} \bar{S}$. This last implication can be rewritten as $S \subset\left\{x \in X: f^{-1}(x) \subset Z\right\} \Longrightarrow \bar{S} \subset\left\{x \in X: f^{-1}(x) \subset Z\right\}$, which makes it clear that the implication holds for all $S$ iff $\left\{x \in X: f^{-1}(x) \subset Z\right\}$ is closed. Therefore (3) is equivalent to (2).

Lemma 13.1.2. Let $f: Y \rightarrow X$ be a continuous, open, surjective map of topological spaces, and let $S$ be a subset of $X$. Then 
(1) The set $S$ is open (respectively, closed, locally closed) in $X$ iff $f^{-1} S$ is open (respectively, closed, locally closed) in $Y$.

(2) Assume further that each fiber of $f$ is an irreducible topological space. Then $S$ is irreducible iff $f^{-1} S$ is irreducible.

Proof. (1) Everything here is well-known (and obvious) except possibly the fact that if $f^{-1} S$ is locally closed, then $S$ is locally closed. So suppose that $f^{-1} S$ is locally closed, which means that $f^{-1} S$ is open in its closure. Using (3) in the previous lemma, we see that $f^{-1} S$ is open in $f^{-1} \bar{S}$. Since the map $f^{-1} \bar{S} \rightarrow \bar{S}$ (obtained by restriction from $f$ ) is obviously open, we conclude that $f f^{-1} S=S$ is open in $\bar{S}$, which means that $S$ is locally closed.

$(2)(\Longleftarrow)$ Clear. $(\Longrightarrow)$ Now assume all fibers of $f$ are irreducible, and assume further that $S$ is irreducible. We must show that $f^{-1} S$ is irreducible, so suppose that $Y_{1}, Y_{2}$ are closed subsets of $Y$ such that $f^{-1} S \subset Y_{1} \cup Y_{2}$. Put $X_{i}:=\{x \in$ $\left.X: f^{-1}(x) \subset Y_{i}\right\}$ for $i=1,2$. We know from the previous lemma that $X_{1}$ and $X_{2}$ are closed in $X$, and using the irreducibility of the fibers of $f$, we see that $S \subset X_{1} \cup X_{2}$. Since $S$ is irreducible, it follows that $S \subset X_{1}$ or $S \subset X_{2}$. Therefore $f^{-1} S \subset f^{-1} X_{1} \subset Y_{1}$ or $f^{-1} S \subset f^{-1} X_{2} \subset Y_{2}$, as desired.

\subsection{Lemma on smooth morphisms.}

Lemma 13.2.1. Let $X, Y$ be schemes locally of finite type over a noetherian base scheme $S$. Let $f: Y \rightarrow X$ be a smooth $S$-morphism. Let $X^{\prime}$ be a locally closed subset of $X$, let $Y^{\prime}$ denote the locally closed subset $f^{-1} X^{\prime}$ of $Y$, and equip both $X^{\prime}$ and $Y^{\prime}$ with their induced reduced subscheme structures. Then the natural morphism $Y^{\prime} \rightarrow Y \times_{X} X^{\prime}$ is an isomorphism, and $Y^{\prime}$ is smooth over $X^{\prime}$. If in addition $X^{\prime} \subset f Y$, then $X^{\prime}$ is smooth over $S$ if and only if $Y^{\prime}$ is smooth over $S$.

Proof. First note that $Y \times_{X} X^{\prime}$ is a subscheme of $Y$ with the same underlying topological space as $Y^{\prime}$. Moreover $Y \times_{X} X^{\prime}$ is smooth over the reduced scheme $X^{\prime}$, and therefore (EGA IV (17.5.7)) $Y \times_{X} X^{\prime}$ is reduced, which implies that $Y^{\prime}=Y \times_{X} X^{\prime}$ as closed subschemes. In particular the morphism $Y^{\prime} \rightarrow X^{\prime}$ is smooth. If in addition $X^{\prime} \subset f Y$, then $Y^{\prime} \rightarrow X^{\prime}$ is also surjective, and it then follows from EGA IV (17.11.1) that $Y^{\prime}$ is smooth over $S$ if and only if $X^{\prime}$ is smooth over $S$. 


\section{Appendix: Some Results of Steinberg}

In [Ste75] Steinberg proves a number of delicate results on the behavior of conjugacy classes in the Lie algebra of $G$ when the characteristic of the base field $k$ is not a torsion prime for $G$. In this paper we are operating under the very strong hypothesis that $|W|$ be invertible in $k$, and this makes life rather simple. Nevertheless it is convenient to obtain what we need as an easy consequence of [Ste75].

14.1. Set-up. Let $S$ be any subset of $\mathfrak{t}$. Define a subset $R_{S}$ of our root system $R$ by

$$
R_{S}:=\{\alpha \in R: \alpha(u)=0 \quad \forall u \in S\} .
$$

Even with no assumption on the characteristic of $k$, it is clear that $R_{S}$ is $\mathbb{Z}$-closed, in the sense that if $\alpha \in R$ lies in the $\mathbb{Z}$-linear span of $R_{S}$ in $X^{*}(T)$, then $\alpha \in R_{S}$. In particular $R_{S}$ is a root system in its own right, whose Weyl group we denote by $W\left(R_{S}\right)$, a subgroup of $W$ which clearly lies inside the subgroup

$$
W_{S}:=\{w \in W: w(u)=u \quad \forall u \in S\} .
$$

Proposition 14.1.1. Assume, as usual, that $|W|$ be invertible in $k$. Then

(1) The subgroups $W_{S}$ and $W\left(R_{S}\right)$ coincide.

(2) The subset $R_{S}$ is $\mathbb{Q}$-closed, in the sense that if $\alpha \in R$ lies in the $\mathbb{Q}$-linear span of $R_{S}$ in $X^{*}(T)$, then $\alpha \in R_{S}$.

(3) There is a Levi subgroup $M \supset T$ in $G$ whose root system $R_{M}$ coincides with $R_{S}$.

Proof. (1) This follows immediately from Corollary 2.8, Lemma 3.7, Corollary 3.11 and Theorem 3.14 in Steinberg's article [Ste75].

(2) Let $L(R)$ (respectively, $L\left(R_{S}\right)$ ) denote the $\mathbb{Z}$-linear span of $R$ (respectively, $\left.R_{S}\right)$ in $X^{*}(T)$. Similarly, let $L\left(R^{\vee}\right)$ (respectively, $L\left(R_{S}^{\vee}\right)$ ) denote the $\mathbb{Z}$-linear span of $R^{\vee}$ (respectively, $R_{S}^{\vee}$ ) in $X_{*}(T)$. Using a suitably normalized $W$-invariant $\mathbb{Z}$ valued symmetric bilinear form on $L\left(R^{\vee}\right)$, we obtain a $W$-equivariant embedding

$$
\varphi: L\left(R^{\vee}\right) \rightarrow L(R)
$$

such that for every $\alpha \in R$ there exists a positive integer $d_{\alpha}$ dividing $|W|$ (hence invertible in $k$ ) such that $\varphi\left(\alpha^{\vee}\right)=d_{\alpha} \alpha$. (In fact we can arrange that $d_{\alpha}$ is always 
1,2 , or 3 , with 3 occurring only when one of the irreducible components of $R$ is of type $G_{2}$.)

Now suppose that $\alpha \in R$ lies in the $\mathbb{Q}$-linear span of $R_{S}$ in $X^{*}(T)$. We must show that $\alpha \in R_{S}$. Since $\varphi$ becomes an isomorphism after tensoring with $\mathbb{Q}$, it is also true that $\alpha^{\vee}$ lies in the $\mathbb{Q}$-linear span of $R_{S}^{\vee}$ in $X_{*}(T)$. Therefore the class of $\alpha^{\vee}$ in $L\left(R^{\vee}\right) / L\left(R_{S}^{\vee}\right)$ is a torsion element, say of order $d$. Any prime $p$ dividing $d$ is a torsion prime for the root system $R$. By Corollary 2.8 of [Ste75] $p$ divides $|W|$, and therefore $p$ is invertible in $k$. We conclude that $d$ is invertible in $k$.

Now $d \alpha^{\vee} \in L\left(R_{S}^{\vee}\right)$, and therefore

$$
d d_{\alpha} \alpha=\varphi\left(d \alpha^{\vee}\right) \in L\left(R_{S}\right)
$$

which implies that $d d_{\alpha} \alpha(u)=0$ for all $u \in S$. Since $d d_{\alpha}$ is invertible in $k$, we conclude that $\alpha(u)=0$ for all $u \in S$, so that $\alpha \in R_{S}$, as desired.

(3) It follows easily from [Bou02, Ch. VI, no. 1.7, Prop. 24] that the $\mathbb{Q}$-closed subsets of $R$ are precisely those of the form $R_{M}$ for some Levi subgroup $M \supset$ $T$.

14.2. A property of $\mathfrak{a}_{M}$. Let $M$ be a Levi subgroup of $G$ containing $T$. Let $R_{M}$ be the set of roots of $T$ in $M$. Define a linear subspace $\mathfrak{a}_{M}$ of $\mathfrak{t}$ by

$$
\mathfrak{a}_{M}:=\left\{u \in \mathfrak{t}: \alpha(u)=0 \quad \forall \alpha \in R_{M}\right\} .
$$

Lemma 14.2.1. Assume, as usual, that $|W|$ be invertible in $k$. Then

$$
R_{M}=\left\{\alpha \in R: \alpha(u)=0 \quad \forall u \in \mathfrak{a}_{M}\right\} .
$$

Proof. Choose a base $B$ for the root system $R$ in such a way that $B \cap R_{M}$ is a base for $R_{M}$. Since the index of connection of $R$ divides $|W|$ (see [Bou02, Ch. VI, no. 2.4, Prop. 7]) and is therefore invertible in $k$, the elements in $B$ yield linearly independent elements of $\mathfrak{t}^{*}$. Note that $\mathfrak{a}_{M}$ is the intersection of the root hyperplanes in $\mathfrak{t}$ determined by the elements in $B \cap R_{M}$.

We must show that if $\alpha \in R \backslash R_{M}$, then $\alpha$ does not vanish identically on $\mathfrak{a}_{M}$. We may assume that $\alpha$ is positive. Inside $X^{*}(T)$ we write $\alpha$ as a $\mathbb{Z}$-linear combination of elements in $B$. Then some element $\beta \in B, \beta \notin R_{M}$ occurs in this linear combination with positive coefficient $n$. It is enough to show that $n$ is non-zero in $k$. This is clear unless $k$ has characteristic $p$ for some prime $p$. 
Let $n^{\prime}$ be the coefficient of $\beta$ in the highest root $\tilde{\alpha}$. Then $n \leq n^{\prime}$, so it is enough to show that $n^{\prime}<p$. This follows from our hypothesis that $|W|$ be invertible in $k$ (check case-by-case).

\section{Appendix: Fixed points of the action of A finite group on A} SCHEME

Throughout this section $G$ denotes a finite group of order $|G|$. For any set $Z$ on which $G$ acts we write $Z^{G}$ for the set of fixed points of the action of $G$ on $Z$. Finally, $S$ denotes some scheme, which will often serve as a base scheme.

15.1. Review of coinvariants of $G$-actions on quasicoherent sheaves. Let $X$ be a scheme and $\mathcal{F}$ a quasicoherent $\mathcal{O}_{X}$-module. We consider an action of $G$ on $\mathcal{F}$, in other words, a homomorphism $\rho: G \rightarrow \operatorname{Aut}_{\mathcal{O}_{X}}(\mathcal{F})$.

We write $\mathcal{F}_{G}$ for the coinvariants of $G$ on $\mathcal{F}$. By definition $\mathcal{F}_{G}$ is the quasicoherent $\mathcal{O}_{X}$-module obtained as the cokernel of the homomorphism

$$
\bigoplus_{g \in G} \mathcal{F} \rightarrow \mathcal{F}
$$

whose restriction to the summand indexed by $g \in G$ is $\rho(g)-\mathrm{id}_{\mathcal{F}}$.

For any $\mathcal{O}_{X}$-module $\mathcal{H}$ there is an obvious action of $G$ on $\operatorname{Hom}_{\mathcal{O}_{X}}(\mathcal{F}, \mathcal{H})$, and it is evident from the definition of coinvariants that there is a canonical isomorphism

$$
\operatorname{Hom}_{\mathcal{O}_{X}}\left(\mathcal{F}_{G}, \mathcal{H}\right)=\left(\operatorname{Hom}_{\mathcal{O}_{X}}(\mathcal{F}, \mathcal{H})\right)^{G}
$$

15.2. Fixed points of $G$-actions on schemes. Let $X$ be a scheme over $S$. Suppose that the finite group $G$ acts on $X$ over $S$, by which we mean that for each $g \in G$ the morphism $x \mapsto g x$ from $X$ to itself is a morphism over $S$.

We define a contravariant set-valued functor $X^{G}$ on the category of schemes $T$ over $S$ by the rule

$$
X^{G}(T):=X(T)^{G}
$$

\section{Lemma 15.2.1.}

(1) The subfunctor $X^{G}$ of $X$ is represented by a locally closed subscheme of $X$.

(2) If $X$ is separated over $S$, then $i: X^{G} \hookrightarrow X$ is a closed immersion. 
(3) If $X$ is locally of finite presentation over $S$, then $X^{G}$ is locally of finite presentation over $S$.

(4) Taking fixed points commutes with arbitrary base change $S^{\prime} \rightarrow S$, which is to say that

$$
\left(X \times{ }_{S} S^{\prime}\right)^{G}=X^{G} \times{ }_{S} S^{\prime} .
$$

Proof. Enumerate the elements of $G$ as $g_{1}, \ldots, g_{n}$. Write $X^{n}$ for the $n$-fold fiber product $X \times_{S} X \times_{S} \cdots \times_{S} X$. We consider two morphisms $X \rightarrow X^{n}$, one being the diagonal morphism $\Delta$ defined by $\Delta(x)=(x, \ldots, x)$, the other, denoted $\alpha$, being defined by $\alpha(x)=\left(g_{1} x, \ldots, g_{n} x\right)$. Taking the fiber product of these two morphisms, we get a scheme over $S$ which clearly represents $X^{G}$.

Thus we have a cartesian square

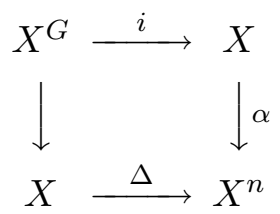

showing that any property of $\Delta$ which is stable under base change will be inherited by $i$. This proves (1), (2) and reduces (3) to checking that $\Delta$ is locally of finite presentation when $X$ is locally of finite presentation over $S$. This follows immediately from EGA IV (1.4.3)(v), applied to the composition $\operatorname{pr}_{1} \circ \Delta$, with $\operatorname{pr}_{1}: X^{n} \rightarrow X$ denoting projection on the first factor.

Finally, (4) is obvious from the definition of $X^{G}$.

15.3. 1-forms over fixed point subschemes. For any scheme $X$ over $S$ one has the quasicoherent $\mathcal{O}_{X}$-module $\Omega_{X / S}^{1}$ of 1 -forms on $X / S$, as well as the tangent "bundle" $T_{X / S}$, which is the scheme, affine over $X$, obtained as the spectrum of the symmetric algebra on the $\mathcal{O}_{X}$-module $\Omega_{X / S}^{1}$.

Consider a morphism $f: Y \rightarrow X$ of schemes over $S$, and a quasicoherent $\mathcal{O}_{Y}$-module $\mathcal{H}$. We regard $\mathcal{O}_{Y} \oplus \mathcal{H}$ as an $\mathcal{O}_{Y}$-algebra in the usual way:

$$
\left(a_{1}, h_{1}\right) \cdot\left(a_{2}, h_{2}\right)=\left(a_{1} a_{2}, a_{1} h_{2}+a_{2} h_{1}\right) .
$$

Put $Y(\mathcal{H}):=\operatorname{Spec}\left(\mathcal{O}_{Y} \oplus \mathcal{H}\right)$, a scheme affine over $Y$. The augmentation $\mathcal{O}_{Y} \oplus \mathcal{H} \rightarrow$ $\mathcal{O}_{Y}$ (sending $(a, h)$ to $a$ ) yields a section of $Y(\mathcal{H}) \rightarrow Y$, which we use to identify $Y$ with a closed subscheme of $Y(\mathcal{H})$ having the same underlying topological space 
as $Y(\mathcal{H})$. We then have (see EGA IV 16.5 ) the following property of $\Omega_{X / S}^{1}$ :

$$
\operatorname{Hom}_{\mathcal{O}_{Y}}\left(f^{*} \Omega_{X / S}^{1}, \mathcal{H}\right)=\left\{\tilde{f} \in \operatorname{Hom}_{S}(Y(\mathcal{H}), X):\left.\tilde{f}\right|_{Y}=f\right\} .
$$

Lemma 15.3.1. Let $X$ be a scheme over $S$, and suppose that the finite group $G$ acts on $X$ over $S$. Let $i: X^{G} \hookrightarrow X$ be the obvious inclusion. Then there are canonical isomorphisms

$$
\Omega_{X^{G} / S}^{1}=\left(i^{*} \Omega_{X / S}^{1}\right)_{G}
$$

and

$$
T_{X^{G} / S}=\left(T_{X / S}\right)^{G} .
$$

The subscript $G$ on the right side of (15.3.2) indicates that we take coinvariants for the action of $G$.

Proof. To prove (15.3.2) it is enough to construct, for any quasicoherent $\mathcal{O}_{X^{G-}}$ module $\mathcal{H}$, a functorial isomorphism

$$
\operatorname{Hom}_{\mathcal{O}_{X} G}\left(\Omega_{X^{G} / S}^{1}, \mathcal{H}\right)=\operatorname{Hom}_{\mathcal{O}_{X} G}\left(\left(i^{*} \Omega_{X / S}^{1}\right)_{G}, \mathcal{H}\right) .
$$

By (15.3.1) we have

$$
\operatorname{Hom}_{\mathcal{O}_{X} G}\left(i^{*} \Omega_{X / S}^{1}, \mathcal{H}\right)=\left\{\tilde{i} \in \operatorname{Hom}_{S}\left(X^{G}(\mathcal{H}), X\right):\left.\tilde{i}\right|_{X^{G}}=i\right\}
$$

Taking invariants under $G$ and using (15.1.1), we see that

$$
\operatorname{Hom}_{\mathcal{O}_{X} G}\left(\left(i^{*} \Omega_{X / S}^{1}\right)_{G}, \mathcal{H}\right)=\left\{\tilde{i} \in \operatorname{Hom}_{S}\left(X^{G}(\mathcal{H}), X^{G}\right):\left.\tilde{i}\right|_{X^{G}}=\operatorname{id}_{X^{G}}\right\},
$$

and by (15.3.1) the right side of this equality is equal to

$$
\operatorname{Hom}_{\mathcal{O}_{X} G}\left(\Omega_{X^{G} / S}^{1}, \mathcal{H}\right)
$$

as desired.

From the definition of $T_{X / S}$ we have, for any scheme $S^{\prime}$ over $S$, the equality

$$
T_{X / S}\left(S^{\prime}\right)=\left\{(f, \beta): f \in \operatorname{Hom}_{S}\left(S^{\prime}, X\right), \beta \in \operatorname{Hom}_{\mathcal{O}_{X}}\left(\Omega_{X / S}^{1}, f_{*} \mathcal{O}_{S^{\prime}}\right)\right\} .
$$

Taking fixed points under $G$, we find that

$$
\left(T_{X / S}\right)^{G}\left(S^{\prime}\right)=\left\{(f, \beta): f \in \operatorname{Hom}_{S}\left(S^{\prime}, X^{G}\right), \beta \in \operatorname{Hom}_{\mathcal{O}_{X}}\left(\Omega_{X / S}^{1}, i_{*} f_{*} \mathcal{O}_{S^{\prime}}\right)^{G}\right\} .
$$

Using (15.3.2), (15.1.1) and the adjointness of $i^{*}, i_{*}$, we see that

$$
\operatorname{Hom}_{\mathcal{O}_{X} G}\left(\Omega_{X^{G} / S}^{1}, f_{*} \mathcal{O}_{S^{\prime}}\right)=\operatorname{Hom}_{\mathcal{O}_{X}}\left(\Omega_{X / S}^{1}, i_{*} f_{*} \mathcal{O}_{S^{\prime}}\right)^{G},
$$


from which it follows that

$$
\begin{aligned}
\left(T_{X / S}\right)^{G}\left(S^{\prime}\right) & =\left\{(f, \beta): f \in \operatorname{Hom}_{S}\left(S^{\prime}, X^{G}\right), \beta \in \operatorname{Hom}_{\mathcal{O}_{X} G}\left(\Omega_{X^{G} / S}^{1}, f_{*} \mathcal{O}_{S^{\prime}}\right)\right\} \\
& =T_{X^{G} / S}\left(S^{\prime}\right),
\end{aligned}
$$

which proves (15.3.3).

15.4. Smoothness of fixed point subschemes. Again consider an action of the finite group $G$ on a scheme $X$ over $S$.

Lemma 15.4.1. Suppose that $X$ is smooth over $S$ and that $|G|$ is invertible on $S$. Then $X^{G}$ is smooth over $S$.

Proof. It follows from Lemma 15.2.1 (3) that $X^{G}$ is locally of finite presentation over $S$. It remains to verify that $X^{G}$ is formally smooth over $S$, so consider an affine scheme $\operatorname{Spec}(A)$ over $S$ and an ideal $I \subset A$ such that $I^{2}=0$. Writing $X^{G}(A)$ for $\operatorname{Hom}_{S}\left(\operatorname{Spec} A, X^{G}\right)$, we must show that

$$
\alpha: X^{G}(A) \rightarrow X^{G}(A / I)
$$

is surjective.

Since $X$ is smooth over $S$, we do know that

$$
\beta: X(A) \rightarrow X(A / I)
$$

is surjective. Given $x \in X(A / I)$, in other words an $S$-morphism $x: \operatorname{Spec} A / I \rightarrow$ $X$, the fiber $\beta^{-1}(x)$ is a principal homogeneous space under (again see EGA IV, $16.5)$

$$
M:=\operatorname{Hom}_{A / I}\left(x^{*} \Omega_{X / S}^{1}, I\right) .
$$

Now suppose that $x \in X^{G}(A / I)$. Then $G$ acts compatibly on $M$ and $\beta^{-1}(x)$, and the obstruction to the existence of a $G$-invariant element in $\beta^{-1}(x)$ lies in $H^{1}(G, M)$. Since $M$ is a $G$-module on which multiplication by $|G|$ is invertible, all higher group cohomology of $M$ vanishes, so our obstruction is automatically trivial. Therefore $\alpha^{-1}(x)$ is non-empty, showing that $\alpha$ is surjective, as desired.

In the next result we no longer need a base scheme $S$. Note that any action of a finite group on a scheme $X$ is automatically an action on $X$ over $\operatorname{Spec}(\mathbb{Z})$, so $X^{G}$ still makes sense and is a scheme (over $\operatorname{Spec}(\mathbb{Z})$ ). 
Lemma 15.4.2. Suppose that the finite group $G$ acts on schemes $X, Y$. Suppose further that we are given a G-equivariant morphism $f: Y \rightarrow X$, and consider the morphism $Y^{G} \rightarrow X^{G}$ induced by $f$.

(1) If $Y$ is locally of finite presentation over $X$, then $Y^{G}$ is locally of finite presentation over $X^{G}$.

(2) There is a canonical isomorphism $\Omega_{Y^{G} / X^{G}}^{1}=\left(i^{*} \Omega_{Y / X}^{1}\right)_{G}$, where $i$ denotes the inclusion $Y^{G} \hookrightarrow Y$ and the subscript $G$ indicates coinvariants.

(3) If $Y$ is smooth over $X$, and $|G|$ is invertible on $X$, then $Y^{G}$ is smooth over $X^{G}$

(4) If $Y \rightarrow X$ is a locally closed immersion, then so is $Y^{G} \rightarrow X^{G}$.

Proof. We already know the first three parts of the lemma when $G$ acts trivially on $X$, so that $X^{G}=X$. To treat the general case, we form the cartesian square

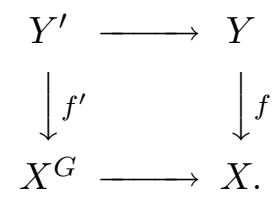

The group $G$ still acts on the locally closed subscheme $Y^{\prime}$ of $Y$, and it is clear that $\left(Y^{\prime}\right)^{G}=Y^{G}$. If $f$ is locally of finite presentation (respectively, smooth), then $f^{\prime}$ is locally of finite presentation (respectively, smooth). Moreover $i^{*} \Omega_{Y / X}^{1}=$ $\left(i^{\prime}\right)^{*} \Omega_{Y^{\prime} / X^{G}}^{1}$, where $i^{\prime}$ is the inclusion $Y^{G} \hookrightarrow Y^{\prime}$. Therefore it is enough to prove the first three parts of the lemma with $f$ replaced by $f^{\prime}$, and then we are done by the remark made at the beginning of the proof.

We now prove the last part of the lemma. Using that $f$ is a monomorphism, we see that the square

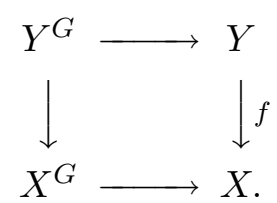

is cartesian, allowing us to deduce that $Y^{G} \rightarrow X^{G}$ is an immersion from the fact that $f$ is an immersion.

16. Appendix. Greenberg's FunCtor

16.1. Definition of Greenberg's functor. Let $X$ be a scheme of finite type over $\mathcal{O}$, and let $N$ be a positive integer. Then Greenberg's functor associates to 
$X$ the scheme $X_{N}$ of finite type over $k$ whose points in any $k$-algebra $A$ are given by

$$
X_{N}(A):=X\left(A \otimes_{k}\left(\mathcal{O} / \epsilon^{N} \mathcal{O}\right)\right) .
$$

In particular the set of $k$-points of $X_{N}$ is $X\left(\mathcal{O} / \epsilon^{N} \mathcal{O}\right)$.

An $\mathcal{O}$-morphism $f: Y \rightarrow X$ between schemes of finite type over $\mathcal{O}$ induces a $k$-morphism

$$
f_{N}: Y_{N} \rightarrow X_{N}
$$

If $f$ is smooth (respectively, étale), then $f_{N}: Y_{N} \rightarrow X_{N}$ is smooth (respectively, étale). Indeed, due to (16.1.1), the formal smoothness (respectively, étaleness) of $f_{N}$ is inherited from the formal smoothness (respectively, étaleness) of $f$.

16.2. The smooth case. Suppose that $X$ is smooth over $\mathcal{O}$. Then $X_{N}$ is smooth over $k$. It follows from (16.1.1) and (15.3.1) that the tangent space to $X_{N}$ at $x \in X_{N}(k)=X\left(\mathcal{O} / \epsilon^{N} \mathcal{O}\right)$ is given by

$$
T_{X_{N}, x}=x^{*} \mathcal{T}_{X / \mathcal{O}}
$$

where $x$ is being regarded as an $\mathcal{O}$-morphism $\operatorname{Spec}\left(\mathcal{O} / \epsilon^{N} \mathcal{O}\right) \rightarrow X$, and $\mathcal{T}_{X / \mathcal{O}}$ is the relative tangent sheaf of $X / \mathcal{O}$. Note that the tangent space $T_{X_{N}, x}$ is in a natural way an $\mathcal{O} / \epsilon^{N} \mathcal{O}$-module, free of finite rank.

Suppose that $x$ is obtained by reduction modulo $\epsilon^{N}$ from $\tilde{x} \in X(\mathcal{O})$. Then $\tilde{x}^{*} \mathcal{T}_{X / \mathcal{O}}$ is a free $\mathcal{O}$-module of finite rank that we will refer to informally as the tangent space to $X(\mathcal{O})$ at $\tilde{x}$ and denote by $T_{X(\mathcal{O}), \tilde{x}}$. Clearly we have

$$
T_{X_{N}, x}=T_{X(\mathcal{O}), \tilde{x}} \otimes_{\mathcal{O}}\left(\mathcal{O} / \epsilon^{N} \mathcal{O}\right) .
$$

Now suppose that $f: Y \rightarrow X$ is an $\mathcal{O}$-morphism between two smooth schemes over $\mathcal{O}$; applying Greenberg's functor to $f$ we get a $k$-morphism

$$
f_{N}: Y_{N} \rightarrow X_{N}
$$

Let $\tilde{y} \in Y(\mathcal{O})$ and put $\tilde{x}:=f(\tilde{y}) \in X(\mathcal{O})$; then let $y \in Y\left(\mathcal{O} / \epsilon^{N} \mathcal{O}\right), x \in$ $X\left(\mathcal{O} / \epsilon^{N} \mathcal{O}\right)$ be the points obtained from $\tilde{y}, \tilde{x}$ by reduction modulo $\epsilon^{N}$. The differential of $f$ gives us an $\mathcal{O}$-linear map

$$
d f_{\tilde{y}}: T_{Y(\mathcal{O}), \tilde{y}} \rightarrow T_{X(\mathcal{O}), \tilde{x}}
$$


Reducing this map modulo $\epsilon^{N}$ and using the isomorphism (16.2.1), we obtain a $k$-linear map

$$
T_{Y_{N}, y} \rightarrow T_{X_{N}, x}
$$

which is easily seen to coincide with the differential of $f_{N}$ at $y$. In other words, the differential of $f_{N}$ is the reduction modulo $\epsilon^{N}$ of the differential of $f$.

16.3. Restriction of scalars $\mathcal{O}_{E} / \mathcal{O}$ and Greenberg's functor. Let $E$ be a finite extension field of $F$, and let $\mathcal{O}_{E}$ be the integral closure of $\mathcal{O}$ in $E$.

Let $X$ be a scheme of finite type over $\mathcal{O}_{E}$. We denote by $R_{\mathcal{O}_{E} / \mathcal{O}} X$ the scheme of finite type over $\mathcal{O}$ obtained by (Weil) restriction of scalars from $\mathcal{O}_{E}$ to $\mathcal{O}$. Recall that the points of $R_{\mathcal{O}_{E} / \mathcal{O}} X$ in any $\mathcal{O}$-algebra $A$ are given by

$$
\left(R_{\mathcal{O}_{E} / \mathcal{O}} X\right)(A)=X\left(A \otimes_{\mathcal{O}} \mathcal{O}_{E}\right) .
$$

Let $N$ be a positive integer. Applying Greenberg's functor to $R_{\mathcal{O}_{E} / \mathcal{O}} X$ provides us with a $k$-scheme whose set of $k$-points is $\left(R_{\mathcal{O}_{E} / \mathcal{O}} X\right)\left(\mathcal{O} / \epsilon^{N} \mathcal{O}\right)=X\left(\mathcal{O}_{E} / \epsilon^{N} \mathcal{O}_{E}\right)$. But there is another equally natural way to produce a $k$-scheme with the same set of $k$-points, namely to apply Greenberg's functor (for the field $E$ rather than the field $F$ ) to $X$ (and the quotient ring $\mathcal{O}_{E} / \epsilon^{N} \mathcal{O}_{E}$ of $\mathcal{O}_{E}$ ). In fact these two $k$-schemes are canonically isomorphic, since for both schemes the set of $A$-valued points ( $A$ now being a $k$-algebra) works out to be

$$
X\left(A \otimes_{k}\left(\mathcal{O}_{E} / \epsilon^{N} \mathcal{O}_{E}\right)\right) .
$$

16.4. Greenberg's functor and fixed point sets. Let $X$ be a scheme of finite type over $\mathcal{O}$, and $N$ a positive integer. From Greenberg's functor we get the $k$ scheme $X_{N}$. Now suppose further that we are given an action of a finite group $G$ on $X$ over $\mathcal{O}$. Then, by functoriality, $G$ acts on $X_{N}$ over $k$.

It follows immediately from the definitions that $\left(X_{N}\right)^{G}$ is canonically isomorphic to $\left(X^{G}\right)_{N}$. Indeed, for both schemes the set of $A$-valued points ( $A$ being a $k$-algebra) works out to be

$$
X\left(A \otimes_{k}\left(\mathcal{O} / \epsilon^{N} \mathcal{O}\right)\right)^{G}
$$

\section{REFERENCES}

[Bez96] R. Bezrukavnikov, The dimension of the fixed point set on affine flag manifolds, Math. Res. Lett. 3 (1996), 185-189. 
[Bou02] N. Bourbaki, Lie groups and Lie algebras. Chapters 4-6, Elements of Mathematics (Berlin), Springer-Verlag, Berlin, 2002.

[GKM06] M. Goresky, R. Kottwitz, and R. MacPherson, Purity of equivalued affine Springer fibers, Represent. Theory 10 (2006), 130-146 (electronic).

[Gre61] M. Greenberg, Schemata over local rings, Ann. of Math. (2) 73 (1961), 624-648.

[Gre63] M. Greenberg, Schemata over local rings. II, Ann. of Math. (2) 78 (1963), 256-266.

[Gre66] M. Greenberg, Rational points in Henselian discrete valuation rings, Inst. Hautes Études Sci. Publ. Math. (1966), no. 31, 59-64.

[Gro67] A. Grothendieck, Éléments de géométrie algébrique. IV. Étude locale des schémas et des morphismes de schémas IV, Inst. Hautes Études Sci. Publ. Math. (1967), no. 32, 361.

[KL88] D. Kazhdan and G. Lusztig, Fixed point varieties on affine flag manifolds, Israel J. Math. 62 (1988), 129-168.

[Spr74] T. Springer, Regular elements of finite reflection groups, Invent. Math. 25 (1974), 159-198.

[Ste75] R. Steinberg, Torsion in reductive groups, Advances in Math. 15 (1975), 63-92.

Mark Goresky and Robert MacPherson

School of Mathematics, Institute for Advanced Study

Princeton, NJ, 08540

E-mail: goresky@ias.edu

E-mail: rdm@math.ias.edu

Robert Kottwitz

Department of Mathematics

University of Chicago

5734 University Avenue

Chicago, Illinois 60637

E-mail: kottwitz@math.uchicago.edu 